This item was submitted to Loughborough's Research Repository by the author.

Items in Figshare are protected by copyright, with all rights reserved, unless otherwise indicated.

\title{
Three-dimensional water-wave scattering in two-layer fluids
}

PLEASE CITE THE PUBLISHED VERSION

LICENCE

CC BY-NC-ND 4.0

\section{REPOSITORY RECORD}

Cadby, Jonathan R., and C.M. Linton. 2019. "Three-dimensional Water-wave Scattering in Two-layer Fluids". figshare. https://hdl.handle.net/2134/741. 


\title{
Three-dimensional water-wave scattering in two-layer fluids
}

\author{
by \\ J.R. Cadby \& C.M. Linton \\ Department of Mathematical Sciences, Loughborough University, Leicestershire LE11 \\ $3 \mathrm{TU}$
}

\begin{abstract}
We consider, using linear water-wave theory, three-dimensional problems concerning the interaction of waves with structures in a fluid which contains a layer of finite depth bounded above by a free surface and below by an infinite layer of fluid of greater density. For such a situation time-harmonic waves can propagate with two different wavenumbers $K$ and $k$. In a single-layer fluid there are a number of reciprocity relations that exist connecting the various hydrodynamic quantities that arise, and these relations are systematically extended to the two-fluid case. The particular problems of wave radiation and scattering by a submerged sphere in either the upper or lower layer are then solved using multipole expansions.
\end{abstract}

\section{Introduction}

This paper represents an extension to three dimensions of the work of Linton and McIver (1995), hereafter referred to as LM, in which a two-dimensional linear scattering theory was developed to study the interaction of water waves with obstacles in fluids consisting of a layer of finite depth bounded above by a free surface and below by an infinite layer of denser fluid. The motivation for this work came from a plan to build an underwater pipe bridge across one of the Norwegian fjords, bodies of water which typically consist of a layer of fresh water about $10 \mathrm{~m}$ thick on top of a very deep body of salt water.

Very little work has been done on wave/structure interactions in such fluid regions, except by approximating the free surface by a rigid lid. With this simplifying assumption Sturova (1994), for example, has studied the radiation of waves by an oscillating cylinder which is also moving uniformly in a direction perpendicular to its axis. With the correct linear free surface boundary condition, but in the absence of obstacles, Lamb (1932) Art. 231 showed that the appropriate dispersion relation for this two-layer configuration has two solutions for a given frequency, one corresponding to waves where the majority of the disturbance is close to the free surface and the other to waves on the interface between the two fluid layers. More recently, Iooss (1999) has studied nonlinear periodic travelling waves in two dimensions, again in the absence of any obstacles.

When a wave is scattered by an obstacle there is the possibility that the wave energy will be transferred between the two possible modes and computations in LM demonstrate this for the case of a submerged horizontal cylinder in either the upper or the lower layer. In this paper we will develop a general three-dimensional linear scattering theory and then illustrate it by solving problems involving submerged spheres. Again the transfer of energy between the two different wave modes will be apparent.

General relations exist between various quantities that arise in water wave radiation and diffraction problems. For a single-layer fluid these can be derived systematically using 
Green's theorem (see, for example, Mei 1983) and they were extended to two-layer fluids in two dimensions in LM. In section 2 below, the equivalent relations are derived for the three-dimensional two-fluid case. The heave and sway radiation problems for a submerged sphere, in either the lower and upper layer, are solved using multipole expansions in section 3 and in section 4 the scattering problem is solved for such bodies.

\section{Three-dimensional radiation and scattering in two- layer fluids}

We take the origin of coordinates in the undisturbed interface between the upper (lighter) fluid and the lower (denser) fluid. Both fluids are assumed to be inviscid and incompressible. Horizontal coordinates are $x$ and $y$ whilst the vertical coordinate $z$ is measured upwards. The undisturbed free surface is $z=d$. Cylindrical polar coordinates defined by

$$
x=R \cos \alpha, \quad y=R \sin \alpha,
$$

are also be used.

The upper fluid, $0<z<d$, is referred to as region $I$, whilst the lower fluid, $z<0$, is region $I I$. The potential in the upper fluid (of density $\rho^{I}$ ) is $\phi^{I}$ and that in the lower fluid (of density $\rho^{I I}>\rho^{I}$ ) is $\phi^{I I}$. The motion is assumed to be irrotational and so both $\phi^{I}$ and $\phi^{I I}$ satisfy Laplace's equation:

$$
\nabla^{2} \phi^{I}=\nabla^{2} \phi^{I I}=0
$$

The ratio of the densities of the two fluids $\rho^{I} / \rho^{I I}(<1)$ will be denoted by $\rho$ and then the linearized boundary conditions on the interface and free surface are

$$
\begin{aligned}
\phi_{z}^{I} & =\phi_{z}^{I I} & & \text { on } z=0, \\
\rho\left(\phi_{z}^{I}-K \phi^{I}\right) & =\phi_{z}^{I I}-K \phi^{I I} & & \text { on } z=0, \\
\phi_{z}^{I} & =K \phi^{I} & & \text { on } z=d,
\end{aligned}
$$

where $K=\omega^{2} / g$, the time-dependence of $\mathrm{e}^{-\mathrm{i} \omega t}$ having been suppressed. The boundary conditions (2.3) and (2.4) represent the continuity of normal velocity and pressure at the interface respectively.

Solutions to these equations which represent waves with wavenumber $u$ can be found provided $u$ is a solution of the dispersion relation, given by

$$
(u-K)\left(K\left(\sigma+\mathrm{e}^{-2 u d}\right)-u\left(1-\mathrm{e}^{-2 u d}\right)\right)=0,
$$

where $\sigma=(1+\rho) /(1-\rho)$. It follows that either $u=K$ or $u=k$, where

$$
K\left(\sigma+\mathrm{e}^{-2 k d}\right)=k\left(1-\mathrm{e}^{-2 k d}\right) .
$$

There is precisely one positive root $k$ which lies in the range

$$
K \sigma<k<\frac{K(\sigma+1)}{1-\mathrm{e}^{-2 K d \sigma}} .
$$


The general form for outgoing cylindrical waves in such a situation can easily be derived. The velocity potential takes a different form in the upper and lower regions which can be characterized by their far-field forms:

$$
\begin{aligned}
\phi_{i}^{I} & \sim\left(\frac{2}{\pi K R}\right)^{1 / 2} \mathrm{e}^{\mathrm{i} K R-\mathrm{i} \pi / 4} \mathrm{e}^{K z} A_{i}(\alpha)+\left(\frac{2}{\pi k R}\right)^{1 / 2} \mathrm{e}^{\mathrm{i} k R-\mathrm{i} \pi / 4} g(z) B_{i}(\alpha) \\
\phi_{i}^{I I} & \sim\left(\frac{2}{\pi K R}\right)^{1 / 2} \mathrm{e}^{\mathrm{i} K R-\mathrm{i} \pi / 4} \mathrm{e}^{K z} A_{i}(\alpha)+\left(\frac{2}{\pi k R}\right)^{1 / 2} \mathrm{e}^{\mathrm{i} k R-\mathrm{i} \pi / 4} \mathrm{e}^{k z} B_{i}(\alpha)
\end{aligned}
$$

as $K r \rightarrow \infty$, where

$$
\begin{aligned}
g(z) & =\frac{K \sigma-k}{K(\sigma-1)} \mathrm{e}^{k z}+\frac{K-k}{K(\sigma-1)} \mathrm{e}^{-k z} \\
& =\frac{\rho^{-1}}{1-\mathrm{e}^{2 k d}} \mathrm{e}^{k z}+\frac{\rho^{-1}-1+\mathrm{e}^{2 k d}}{1-\mathrm{e}^{2 k d}} \mathrm{e}^{-k z}
\end{aligned}
$$

An incident plane wave with wavenumber $k$ making an angle $\alpha_{\text {inc }}$ with the positive $x$-axis has the form

$$
\begin{aligned}
& \phi_{\text {inc }}^{I}=g(z) \mathrm{e}^{\mathrm{i} k R \cos \left(\alpha-\alpha_{\mathrm{inc}}\right)} \\
& \phi_{\text {inc }}^{I I}=\mathrm{e}^{k z} \mathrm{e}^{\mathrm{i} k R \cos \left(\alpha-\alpha_{\mathrm{inc}}\right)},
\end{aligned}
$$

whereas an incident wave of wavenumber $K$ has the form

$$
\phi_{\text {inc }}=\mathrm{e}^{K z} \mathrm{e}^{\mathrm{i} K R \cos \left(\alpha-\alpha_{\text {inc }}\right)}
$$

in both the upper and lower fluids.

Now consider a situation in which there are a number of bodies, some in the upper layer, some in the lower layer, and some straddling the two. The boundaries of those bodies in the upper fluid will be denoted by $B^{I}$ and those in the lower fluid by $B^{I I}$. Assume that $\phi$ and $\psi$ are solutions to two different problems, both satisfying (2.2)-(2.5) with $\partial \phi / \partial n$ and $\partial \psi / \partial n$ given on the boundaries $B^{I}$ and $B^{I I}$. If we apply Green's theorem we obtain

$$
\int_{S}\left(\phi \frac{\partial \psi}{\partial n}-\psi \frac{\partial \phi}{\partial n}\right) \mathrm{d} s=0
$$

since $\phi$ and $\psi$ are harmonic functions. Here $S$ is the boundary of a fluid region completely contained in one of the fluid layers and $\partial / \partial n$ is the derivative with respect to the outward normal. In region $I, S$ is composed of the free surface, the boundary of the bodies $B^{I}$, the interface and a cylinder $S_{\infty}^{I}$ whose radius is sufficiently large for the asymptotic forms of the potentials to be used. In region $I I, S$ is composed of a surface parallel to the interface whose depth will be made to tend to infinity, the boundary of the bodies $B^{I I}$, the interface and a cylinder of large radius $S_{\infty}^{I I}$.

It follows from (2.3) and (2.4) that

$$
\rho\left(\phi^{I} \frac{\partial \psi^{I}}{\partial z}-\psi^{I} \frac{\partial \phi^{I}}{\partial z}\right)=\phi^{I I} \frac{\partial \psi^{I I}}{\partial z}-\psi^{I I} \frac{\partial \phi^{I I}}{\partial z} \quad \text { on } z=0 .
$$


If we apply Green's theorem in region $I$ and in region $I I$ the resulting formulas can be added so that the integral along the interface vanishes because of (2.17). This yields

$$
\begin{aligned}
\rho \int_{B^{I}}\left(\phi^{I} \frac{\partial \psi^{I}}{\partial n}\right. & \left.-\psi^{I} \frac{\partial \phi^{I}}{\partial n}\right) \mathrm{d} s+\int_{B^{I I}}\left(\phi^{I I} \frac{\partial \psi^{I I}}{\partial n}-\psi^{I I} \frac{\partial \phi^{I I}}{\partial n}\right) \mathrm{d} s \\
& =-\rho \int_{S_{\infty}^{I}}\left(\phi^{I} \frac{\partial \psi^{I}}{\partial n}-\psi^{I} \frac{\partial \phi^{I}}{\partial n}\right) \mathrm{d} s-\int_{S_{\infty}^{I I}}\left(\phi^{I I} \frac{\partial \psi^{I I}}{\partial n}-\psi^{I I} \frac{\partial \phi^{I I}}{\partial n}\right) \mathrm{d} s
\end{aligned}
$$

By using this equation with radiation and scattering potentials for $\phi$ and $\psi$ we can obtain a number of relations between various hydrodynamic quantities.

\subsection{Two Radiation Potentials}

First we consider the case of two radiation potentials. Let $\phi=\phi_{i}$ and $\psi=\phi_{j}$ be two radiation potentials whose behaviour in the far field is given by (2.9) and (2.10) and which satisfy the body boundary conditions

$$
\frac{\partial \phi_{i}}{\partial n}=n_{i}, \quad \frac{\partial \phi_{j}}{\partial n}=n_{j} \quad \text { on } S_{B}
$$

where $n_{i}$ is the component of the inward normal to the body in the $i$-th mode of motion and $S_{B}$ is the body boundary, which for simplicity we will assume is entirely contained within region I or region II. Now, using (2.7) we can show that

$$
\rho \int_{0}^{d} \mathrm{e}^{K z} g(z) \mathrm{d} z=-\int_{-\infty}^{0} \mathrm{e}^{(K+k) z} \mathrm{~d} z
$$

and it follows that the right-hand side of (2.18) is zero leaving

$$
\int_{S_{B}}\left(\phi_{i} n_{j}-\phi_{j} n_{i}\right) \mathrm{d} s=0 .
$$

The added-mass and damping matrices, $\boldsymbol{\mu}$ and $\boldsymbol{\nu}$ respectively, are real and defined by

$$
-\mathrm{i} \omega \mu_{i j}+\nu_{i j}=-\mathrm{i} \omega \rho^{I I} \delta \int_{S_{B}} \phi_{i} n_{j} \mathrm{~d} s,
$$

where $\delta=\rho$ if the bodies are in fluid $I$ or $\delta=1$ if they are in fluid $I I$. Thus (2.21) states that the added mass and damping matrices are symmetric.

Suppose now we use $\psi=\overline{\phi_{j}}$. The function $\overline{\phi_{j}}$ satisfies the conjugate of the equations governing $\phi_{j}$ and describes an incoming cylindrical wave far from the body. Thus, using the fact that the $n_{i}$ are real, (2.18) becomes

$$
\delta \int_{S_{B}}\left(\phi_{i} n_{j}-\overline{\phi_{j}} n_{i}\right) \mathrm{d} s=\frac{2 \mathrm{i}}{\pi}\left(J_{K} \int_{0}^{2 \pi} A_{i}(\alpha) \overline{A_{j}}(\alpha) \mathrm{d} \alpha+J_{k} \int_{0}^{2 \pi} B_{i}(\alpha) \overline{B_{j}}(\alpha) \mathrm{d} \alpha\right),
$$

where

$$
J_{K}=\frac{1}{K}+2 \rho \int_{0}^{d} \mathrm{e}^{2 K z} \mathrm{~d} z, \quad J_{k}=\frac{1}{k}+2 \rho \int_{0}^{d}[g(z)]^{2} \mathrm{~d} z .
$$

In particular

$$
\nu_{i i}=\frac{\rho^{I I} \omega}{\pi}\left[J_{K} \int_{0}^{2 \pi}\left|A_{i}(\alpha)\right|^{2} \mathrm{~d} \alpha+J_{k} \int_{0}^{2 \pi}\left|B_{i}(\alpha)\right|^{2} \mathrm{~d} \alpha\right],
$$

showing that the diagonal elements of the damping coefficient matrix are proportional to the energy radiated away from the body. 


\section{$2.2 \quad$ A radiation and a scattering potential}

Suppose now we use a scattering potential $\phi=\phi_{\text {inc }}+\phi_{S}$ (i.e. $\partial \phi / \partial n=0$ on the body boundary) and a radiation potential $\psi=\phi_{i}$, where $\phi_{\text {inc }}$ is an incident plane wave. Since both $\phi_{i}$ and $\phi_{S}$ are outgoing cylindrical waves in the far field, the contribution to the right-hand side of (2.18) from the products of these terms will be zero in exactly the same way as when we considered two radiation potentials above. Thus (2.18) becomes

$$
\begin{aligned}
\delta \int_{S_{B}} \phi n_{i} \mathrm{~d} s=\lim _{K R \rightarrow \infty}\left[-\rho \int_{0}^{d} \int_{0}^{2 \pi}\right. & \left(\phi_{\mathrm{inc}}^{I} \frac{\partial \phi_{i}^{I}}{\partial R}-\phi_{i}^{I} \frac{\partial \phi_{\mathrm{inc}}^{I}}{\partial R}\right) R \mathrm{~d} \theta \mathrm{d} z \\
& \left.-\int_{-\infty}^{0} \int_{0}^{2 \pi}\left(\phi_{\mathrm{inc}}^{I I} \frac{\partial \phi_{i}^{I I}}{\partial R}-\phi_{i}^{I I} \frac{\partial \phi_{\mathrm{inc}}^{I I}}{\partial R}\right) R \mathrm{~d} \theta \mathrm{d} z\right] .
\end{aligned}
$$

If we consider the case of an incident wave of wavenumber $K$ making an angle $\alpha_{\text {inc }}$ with the positive $x$-axis we obtain

$$
\begin{aligned}
\delta \int_{S_{B}} \phi n_{i} \mathrm{~d} s= & \lim _{K R \rightarrow \infty}\left[-\mathrm{i} J_{K}\left(\frac{K R}{2 \pi}\right)^{1 / 2} \mathrm{e}^{-\mathrm{i} \pi / 4}\right. \\
& \left.\times \int_{0}^{2 \pi}\left(1-\cos \left(\alpha-\alpha_{\text {inc }}\right)\right) \mathrm{e}^{\mathrm{i} K R\left(1+\cos \left(\alpha-\alpha_{\mathrm{inc}}\right)\right)} A_{i}(\alpha) \mathrm{d} \alpha\right] \\
= & -2 \mathrm{i} J_{K} A_{i}\left(\alpha_{\mathrm{inc}}+\pi\right),
\end{aligned}
$$

the last step being justified through stationary phase arguments. For an incident wave of wavenumber $k$ similar arguments reveal that

$$
\delta \int_{S_{B}} \phi n_{i} \mathrm{~d} s=-2 \mathrm{i} J_{k} B_{i}\left(\alpha_{\text {inc }}+\pi\right) .
$$

The hydrodynamic force on the body in the $i^{t h}$ mode of motion can be written $F_{i}(t)=$ $\operatorname{Re}\left\{f_{i} \mathrm{e}^{-\mathrm{i} \omega t}\right\}$ where $f_{i}$ is found by integrating the dynamic pressure times the appropriate component of the normal over the body surface. In other words

$$
f_{i}=\mathrm{i} \rho^{I I} \delta \omega \int_{S_{B}} \phi n_{i} \mathrm{~d} s
$$

which when combined with (2.28) gives the exciting force in the $i$-th direction due to an incident wave of wavenumber $K$ as

$$
f_{i}=2 \rho^{I I} \omega J_{K} A_{i}\left(\alpha_{\mathrm{inc}}+\pi\right)
$$

When the incident wave has wavenumber $k$ we obtain

$$
f_{i}=2 \rho^{I I} \omega J_{k} B_{i}\left(\alpha_{\mathrm{inc}}+\pi\right)
$$

In both cases the exciting force is related to the amplitude of the radiated wave with the same wavenumber as the incident wave in the direction opposite to that of the incident wave. These formulas represent extensions to two-layer fluids of the Haskind relations (see Newman 1976). 


\subsection{Two scattering potentials}

If $\phi$ and $\psi$ in (2.18) are both scattering potentials then the body surface integrals will be zero leaving

$$
-\rho \int_{S_{\infty}^{I}}\left(\phi^{I} \frac{\partial \psi^{I}}{\partial n}-\psi^{I} \frac{\partial \phi^{I}}{\partial n}\right) \mathrm{d} s-\int_{S_{\infty}^{I I}}\left(\phi^{I I} \frac{\partial \psi^{I I}}{\partial n}-\psi^{I I} \frac{\partial \phi^{I I}}{\partial n}\right) \mathrm{d} s=0 .
$$

We will use the notation $\phi\left(u, \alpha_{i}\right)$ to represent a scattering potential for which the incident wave has wavenumber $u$ and makes an angle $\alpha_{i}$ with the positive $x$-axis. In the far field such a potential takes the form

$$
\begin{gathered}
\phi^{I}\left(u, \alpha_{i}\right) \sim \phi_{\text {inc }}^{I}+\left(\frac{2}{\pi K R}\right)^{1 / 2} \mathrm{e}^{\mathrm{i} K R-\mathrm{i} \pi / 4} \mathrm{e}^{K z} A_{u}^{(i)}(\alpha)+\left(\frac{2}{\pi k R}\right)^{1 / 2} \mathrm{e}^{\mathrm{i} k R-\mathrm{i} \pi / 4} g(z) B_{u}^{(i)}(\alpha) \\
\phi^{I I}\left(u, \alpha_{i}\right) \sim \phi_{\text {inc }}^{I I}+\left(\frac{2}{\pi K R}\right)^{1 / 2} \mathrm{e}^{\mathrm{i} K R-\mathrm{i} \pi / 4} \mathrm{e}^{K z} A_{u}^{(i)}(\alpha)+\left(\frac{2}{\pi k R}\right)^{1 / 2} \mathrm{e}^{\mathrm{i} k R-\mathrm{i} \pi / 4} \mathrm{e}^{k z} B_{u}^{(i)}(\alpha)
\end{gathered}
$$

with the appropriate form for the incident wave depending on its wavenumber.

Using (2.33) with $\phi=\phi\left(K, \alpha_{1}\right)$ and $\psi=\phi\left(K, \alpha_{2}\right)$ we obtain

$$
A_{K}^{(2)}\left(\alpha_{1}+\pi\right)=A_{K}^{(1)}\left(\alpha_{2}+\pi\right)
$$

and with $\phi=\phi\left(k, \alpha_{1}\right)$ and $\psi=\phi\left(k, \alpha_{2}\right)$ we get

$$
B_{k}^{(2)}\left(\alpha_{1}+\pi\right)=B_{k}^{(1)}\left(\alpha_{2}+\pi\right) .
$$

In other words, if we have two incident waves of wavenumber $K(k)$ the amplitude of the first scattered wave with wavenumber $K(k)$ towards the second incident wave is the same as that of the second scattered wave towards the first. With $\phi=\phi\left(K, \alpha_{1}\right)$ and $\psi=\phi\left(k, \alpha_{2}\right),(2.18)$ reduces to

$$
A_{k}^{(2)}\left(\alpha_{1}+\pi\right)=J B_{K}^{(1)}\left(\alpha_{2}+\pi\right)
$$

where $J=J_{k} / J_{K}$. Equations (2.36)-(2.38) are the three-dimensional analogues of equations (2.27)-(2.32) from LM.

The complex conjugate of a scattering potential satisfies the same condition on the body boundary and so (2.18) still applies if either or both of $\phi$ and $\psi$ are of this form. With $\phi=\phi\left(K, \alpha_{1}\right)$ and $\psi=\bar{\phi}\left(K, \alpha_{2}\right)$ we obtain, after considerable algebra,

$$
\pi \overline{A_{K}^{(2)}}\left(\alpha_{1}\right)+\pi A_{K}^{(1)}\left(\alpha_{2}\right)+\int_{0}^{2 \pi} A_{K}^{(1)}(\alpha) \overline{A_{K}^{(2)}}(\alpha) \mathrm{d} \alpha+J \int_{0}^{2 \pi} B_{K}^{(1)}(\alpha)_{K}^{\overline{(2)}}(\alpha) \mathrm{d} \alpha=0
$$

and with $\phi=\phi\left(k, \alpha_{1}\right)$ and $\psi=\bar{\phi}\left(k, \alpha_{2}\right)$ the result is

$$
\pi J \overline{B_{k}^{(2)}}\left(\alpha_{1}\right)+\pi J B_{k}^{(1)}\left(\alpha_{2}\right)+\int_{0}^{2 \pi} B_{k}^{(1)}(\alpha) \overline{B_{k}^{(2)}}(\alpha) \mathrm{d} \alpha+J \int_{0}^{2 \pi} B_{k}^{(1)}(\alpha) \overline{B_{k}^{(2)}}(\alpha) \mathrm{d} \alpha=0 .
$$

Finally, with $\phi=\phi\left(K, \alpha_{1}\right)$ and $\psi=\bar{\phi}\left(k, \alpha_{2}\right)$ we get

$$
\pi \overline{A_{k}^{(2)}}\left(\alpha_{1}\right)+\pi J B_{K}^{(1)}\left(\alpha_{2}\right)+\int_{0}^{2 \pi} A_{K}^{(1)}(\alpha) \overline{A_{k}^{(2)}}(\alpha) \mathrm{d} \alpha+J \int_{0}^{2 \pi} B_{K}^{(1)}(\alpha) \overline{B_{k}^{(2)}}(\alpha) \mathrm{d} \alpha=0 .
$$


These last three relations are the three-dimensional analogues of equations (2.38)-(2.43) from LM.

To complete the reciprocity relations the two-layer equivalent of the three-dimensional Bessho-Newman relations can be derived. The potential $\phi_{i}-\bar{\phi}_{i}$, where $\phi_{i}$ is a radiation potential whose behaviour in the far field is given by (2.9) and (2.10) and which satisfies the body boundary condition given in (2.19), has zero normal derivative on the body boundary (since $n_{i}$ is real) and is thus an appropriate potential to use in (2.33). We apply (2.33) to the potential $\phi=\phi_{i}-\overline{\phi_{i}}$ and each of the scattering potentials $\psi=\phi\left(K, \alpha_{\text {inc }}\right)$ (for which the scattering amplitudes will be labelled $A_{K}(\alpha)$ and $\left.B_{K}(\alpha)\right)$ and $\psi=\phi\left(k, \alpha_{\text {inc }}\right)$ (for which the scattering amplitudes will be labelled $A_{k}(\alpha)$ and $B_{k}(\alpha)$ ) in turn. The three-dimensional equivalents of LM, equations (2.69)-(2.72), can thus be shown to be

$$
\pi A_{i}\left(\alpha_{\mathrm{inc}}+\pi\right)+\pi \overline{A_{i}}\left(\alpha_{\mathrm{inc}}\right)+\int_{0}^{2 \pi} A_{K}(\alpha) \overline{A_{i}}(\alpha) \mathrm{d} \alpha+J \int_{0}^{2 \pi} B_{K}(\alpha) \overline{B_{i}}(\alpha) \mathrm{d} \alpha=0
$$

and

$$
\int_{0}^{2 \pi} A_{k}(\alpha) \overline{A_{i}}(\alpha) \mathrm{d} \alpha+J\left(\pi B_{i}\left(\alpha_{\mathrm{inc}}+\pi\right)+\pi \overline{B_{i}}\left(\alpha_{\mathrm{inc}}\right)+\int_{0}^{2 \pi} B_{k}(\alpha) \overline{B_{i}}(\alpha) \mathrm{d} \alpha\right)=0 .
$$

\section{Radiation problems for a submerged sphere}

To illustrate the general theory of three-dimensional wave/structure interactions in twolayer fluids we will solve various problems involving submerged spheres. Radiation and scattering problems for such geometries can be solved using multipole expansions, the technique having been used to solve similar problems in unstratified fluids by Srokosz (1979) (deep water) and Linton (1991) (finite depth). The centre of the sphere will be at $x=y=0, z=f$ so that if $f<0$ the sphere is in the lower layer, whereas if $f>0$ the sphere is in the upper layer. We will use spherical coordinates $(r, \theta, \alpha)$ centred on the sphere defined by

$$
x=r \sin \theta \cos \alpha, \quad y=r \sin \theta \sin \alpha, \quad z-f=r \cos \theta,
$$

with $r=a$ being the sphere surface. If $f<0$ we require $a<|f|$, whereas if $f>0$ we need $a<\min (d-f, f)$.

\subsection{Sphere in lower fluid layer}

In the present context, multipoles are solutions of the governing equation which are singular at the centre of the sphere, satisfy all the boundary conditions of the problem except that on the sphere surface and behave like outgoing cylindrical waves at large horizontal distances from the singularity. These can be constructed using the method devised by Thorne (1953). A solution of Laplace's equation singular at $z=f$ is $r^{-n-1} P_{n}^{m}(\cos \theta) \cos m \alpha, n \geq m \geq 0$, and this has the integral representation, valid for $z>f$,

$$
\frac{P_{n}^{m}(\cos \theta)}{r^{n+1}} \cos m \alpha=\frac{\cos m \alpha}{(n-m) !} \int_{0}^{\infty} u^{n} \mathrm{e}^{-u(z-f)} J_{m}(u R) \mathrm{d} u .
$$

Note that the definition of $P_{n}^{m}$ used here corresponds to that in Thorne's paper: $P_{n}^{m}(\cos \theta)=$ $\sin ^{m} \theta \mathrm{d}^{m} P_{n}(\cos \theta) / \mathrm{d}(\cos \theta)^{m}$, which differs by a factor of $(-1)^{m}$ from that used by some 
other authors. We look for solutions to $(2.2)-(2.5)$ in the form $\phi_{n}^{m} \cos m \alpha$ where (in an obvious notation)

$$
\begin{aligned}
\phi_{n}^{I m} & =\frac{a^{n+1}}{(n-m) !} \int_{0}^{\infty} u^{n}\left[A_{L}(u) \mathrm{e}^{u z}+B_{L}(u) \mathrm{e}^{-u z}\right] J_{m}(u R) \mathrm{d} u \\
\phi_{n}^{I I m} & =\left(\frac{a}{r}\right)^{n+1} P_{n}^{m}(\cos \theta)+\frac{a^{n+1}}{(n-m) !} \int_{0}^{\infty} u^{n} C_{L}(u) \mathrm{e}^{u z} J_{m}(u R) \mathrm{d} u,
\end{aligned}
$$

and in order to satisfy the boundary conditions on the interface and free surface we find, exactly as in LM, that

$$
\begin{aligned}
& A_{L}(u)=K(1+\sigma)(u+K) \mathrm{e}^{u(f-2 d)} /(u-K) h(u), \\
& B_{L}(u)=K(1+\sigma) \mathrm{e}^{u f} / h(u), \\
& C_{L}(u)=(u+K) \mathrm{e}^{u f}\left[(u+K \sigma) \mathrm{e}^{-2 u d}-u+K\right] /(u-K) h(u),
\end{aligned}
$$

where

$$
h(u)=(u+K) \mathrm{e}^{-2 u d}-u+K \sigma .
$$

The function $h$ is zero at $u=k$, from (2.7), and so the integrands in (3.3) and (3.4) have poles at $u=K$ and $u=k$. In order that the multipoles behave like outgoing waves as $R \rightarrow \infty$, the path of integration is indented to pass beneath these two poles.

The far-field form of $\phi_{n}^{m}$, in the lower fluid, is then

$$
\phi_{n}^{I I m} \sim-\frac{(-\mathrm{i})^{m+1} a^{n+1}}{(n-m) !}\left(\frac{2 \pi}{R}\right)^{1 / 2}\left(K^{n-1 / 2} \mathrm{e}^{\mathrm{i} K R} C_{L}^{K} \mathrm{e}^{K z}+k^{n-1 / 2} \mathrm{e}^{\mathrm{i} k R} C_{L}^{k} \mathrm{e}^{k z}\right) \mathrm{e}^{-\mathrm{i} \pi / 4}
$$

as $K R \rightarrow \infty$, where $C_{L}^{K}$ and $C_{L}^{k}$ are the residues of $C_{L}(u)$ at $u=K$ and $u=k$ respectively, which are given by

$$
C_{L}^{K}=\frac{2 K(1+\sigma) \mathrm{e}^{K(f-2 d)}}{2 \mathrm{e}^{-2 K d}-1+\sigma}
$$

and

$$
C_{L}^{k}=\frac{(K+k) \mathrm{e}^{k f}\left[(K \sigma+k) \mathrm{e}^{-2 k d}-k+K\right]}{(k-K)\left[(1-2 d(K+k)) \mathrm{e}^{-2 k d}-1\right]} .
$$

The multipoles defined by (3.3) and (3.4) can be expanded about $r=0$ in spherical coordinates by using the identity (see Thorne 1953)

$$
\mathrm{e}^{ \pm u(z-f)} J_{m}(u R)=( \pm 1)^{m} \sum_{s=m}^{\infty} \frac{( \pm u r)^{s}}{(s+m) !} P_{s}^{m}(\cos \theta)
$$

This gives

$$
\phi_{n}^{I I m}=\left(\frac{a}{r}\right)^{n+1} P_{n}^{m}(\cos \theta)+\sum_{s=m}^{\infty} A_{n s}^{m}\left(\frac{r}{a}\right)^{s} P_{s}^{m}(\cos \theta)
$$


where

$$
A_{n s}^{m}=\frac{a}{(n-m) !(s+m) !} f_{0}^{\infty}(a u)^{n+s} \mathrm{e}^{u f} C_{L}(u) \mathrm{d} u .
$$

For computational purposes we note that the contour integral in the above expression can be written

$$
f_{0}^{\infty}(a u)^{n+s} \mathrm{e}^{u f} C_{L}(u) \mathrm{d} u+\pi \mathrm{i}(K a)^{n+s} \mathrm{e}^{K f} C_{L}^{K}+\pi \mathrm{i}(k a)^{n+s} \mathrm{e}^{k f} C_{L}^{k}
$$

and that the principal-value integral can easily be evaluated using the method described in Linton and Evans (1992), section 2.

The solutions to the problems of heave and sway will be denoted by $\phi^{0}$ and $\phi^{1}$ respectively. In the case of heave the body velocity is given by $U^{0}=R e\left\{U \mathrm{e}^{-\mathrm{i} w t}\right\} \mathbf{k}$, where $\mathbf{k}$ is a unit vector in the $z$-direction, whereas in sway the body velocity is $\boldsymbol{U}^{1}=\operatorname{Re}\left\{U \mathrm{e}^{-\mathrm{i} w t}\right\} \mathbf{i}$, where $\mathbf{i}$ is a unit vector in the $x$-direction. Hence, since $P_{1}^{0}(\cos \theta)=\cos \theta$ and $P_{1}^{1}(\cos \theta)=$ $\sin \theta$, we have the following body boundary conditions for the heave and sway problems:

$$
\frac{\partial \phi^{m}}{\partial r}=U P_{1}^{m}(\cos \theta) \cos m \alpha \quad \text { on } \quad r=a, \quad 0 \leq \theta \leq \pi, \quad 0 \leq \alpha<2 \pi, \quad m=0,1 .
$$

An appropriate multipole expansion for the velocity potential $\phi^{m}$ is

$$
\phi^{m}=U a \cos m \alpha \sum_{n=1}^{\infty} b_{n}^{m} \phi_{n}^{m} \quad m=0,1,
$$

for some unknown coefficients $b_{n}^{m}$. Note that the $n=0$ term which could appear in the expansion for $\phi^{0}$ has been omitted since this term corresponds to a point source, which is physically unacceptable as it would imply an instantaneous flux of fluid across the surface of the sphere.

The expansion (3.16) satisfies all the conditions of the problem except that on the body surface, (3.15). By applying this condition and using orthogonality relations for associated Legendre functions (Abramowitz and Stegun 1965, eqns 8.4.11 \& 8.4.13) we obtain the infinite system of linear equations

$$
b_{s}^{m}-\frac{s}{s+1} \sum_{n=1}^{\infty} A_{n s}^{m} b_{n}^{m}=-\frac{\delta_{1 s}}{2} \quad s \geq 1, \quad m=0,1,
$$

where $\delta_{n s}$ is the Kronecker delta, for the unknown coefficients $b_{n}^{m}$. This system can be solved numerically by truncating it to an $N \times N$ system and increasing $N$ until the solution converges to the required degree of accuracy. One of the advantages of the multipole method for solving problems of this type is that the truncation parameter required to achieve accurate results is very small; in the computations presented below a value of $N=4$ was used.

The added-mass and damping coefficients $\mu$ and $\nu$ (the diagonal entries in the addedmass and damping matrices, non-dimensionalized with respect to the mass of the fluid displaced by the sphere, $M$, and the maximum acceleration of the sphere, $U \omega$ ) are given by $(2.22)$ as

$$
\mu^{m}+\mathrm{i} \nu^{m}=-\frac{\rho^{I I}}{M U} \int_{0}^{2 \pi} \int_{0}^{\pi} \phi^{m}(a, \theta, \alpha) P_{1}^{m}(\cos \theta) \cos m \alpha a^{2} \sin \theta \mathrm{d} \theta \mathrm{d} \alpha \quad m=0,1 .
$$


Using (3.13) and (3.16), we can reduce this to

$$
\mu^{m}+\mathrm{i} \nu^{m}=-b_{1}^{m}-\sum_{n=1}^{\infty} A_{n 1}^{m} b_{n}^{m}
$$

which can be further simplified by using (3.17) with $r=1$ to give

$$
\mu^{m}+\mathrm{i} \nu^{m}=-1-3 b_{1}^{m} \quad m=0,1 .
$$

The far field form for $\phi^{m}$, in the lower fluid layer, can be written

$$
U^{-1} \phi^{m} \sim\left(\frac{2}{\pi K R}\right)^{1 / 2} \mathrm{e}^{\mathrm{i} K R-\mathrm{i} \pi / 4} \mathrm{e}^{K z} A^{m}(\alpha)+\left(\frac{2}{\pi k R}\right)^{1 / 2} \mathrm{e}^{\mathrm{i} k R-\mathrm{i} \pi / 4} \mathrm{e}^{k z} B^{m}(\alpha)
$$

as $K R \rightarrow \infty$ and from (3.9) and (3.16) we have that

$$
\begin{aligned}
\left|A^{m}(\alpha)\right|^{2} & =\left|\sum_{n=1}^{\infty} \frac{(K a)^{n} b_{n}^{m}}{(n-m) !}\right|^{2}\left(\pi a^{2} C_{L}^{K} \cos m \alpha\right)^{2} \\
\left|B^{m}(\alpha)\right|^{2} & =\left|\sum_{n=1}^{\infty} \frac{(k a)^{n} b_{n}^{m}}{(n-m) !}\right|^{2}\left(\pi a^{2} C_{L}^{k} \cos m \alpha\right)^{2} .
\end{aligned}
$$

Any numerical results produced by solving (3.17) can then be checked against the identity

$$
\operatorname{Im}\left(b_{1}^{m}\right)=-\frac{\pi a}{4}\left(J_{K}\left|C_{L}^{K} \sum_{n=1}^{\infty} \frac{(K a)^{n} b_{n}^{m}}{(n-m) !}\right|^{2}+J_{k}\left|C_{L}^{k} \sum_{n=1}^{\infty} \frac{(k a)^{n} b_{n}^{m}}{(n-m) !}\right|^{2}\right)
$$

which follows by computing the left- and right-hand sides of (2.25) using (3.20) and (3.22)-(3.23) respectively.

Curves of added-mass and damping coefficients for spheres in the lower fluid layer in both heave and sway are shown in figures $1-4$. In all the curves $\rho\left(=\rho^{I} / \rho^{I I}\right)$ is 0.95 and the ratio of the depth of the upper fluid layer to the radius of sphere, $d / a$, is 2.0. Each plot shows the results obtained for four different submersion depths, $f / a$, of the sphere, $-1.1,-1.5,-2$ and -3 . The case $f / a=-1.1$ represents a sphere close to the interface between the two fluid layers, with $|f / a|$ increasing as the sphere becomes more deeply submerged.

Figures 1 and 2 show the damping coefficients for heave and sway respectively and it can be seen that in each case there are two local maximums. These occur near $k a=1$, which corresponds to $K a \approx 0.025$, and $K a=1$ and in order to satisfactorily illustrate both of them on the same figure we have plotted the results (here and subsequently) on a log scale. The damping coefficient is proportional to the radiated energy, see (2.25), and the local maximum around $k a=1$ corresponds to the sphere's increased ability to make waves on the interface near this frequency, whereas the local maximum around $K a=1$ corresponds to the sphere's increased free surface wave-making ability (see, for example, Linton 1991). As one would expect, the closer the sphere is to the interface the greater the wave-making capability and hence the greater the damping coefficient. Since the sphere is in the lower layer it affects the interface a lot more than the free surface and so the variation in the damping coefficient near $k a=1$ is greater than that near $K a=1$. In all cases the heave damping coefficient is greater than the sway damping coefficient, just as was reported by Srokosz (1979) for the single-layer fluid case. 


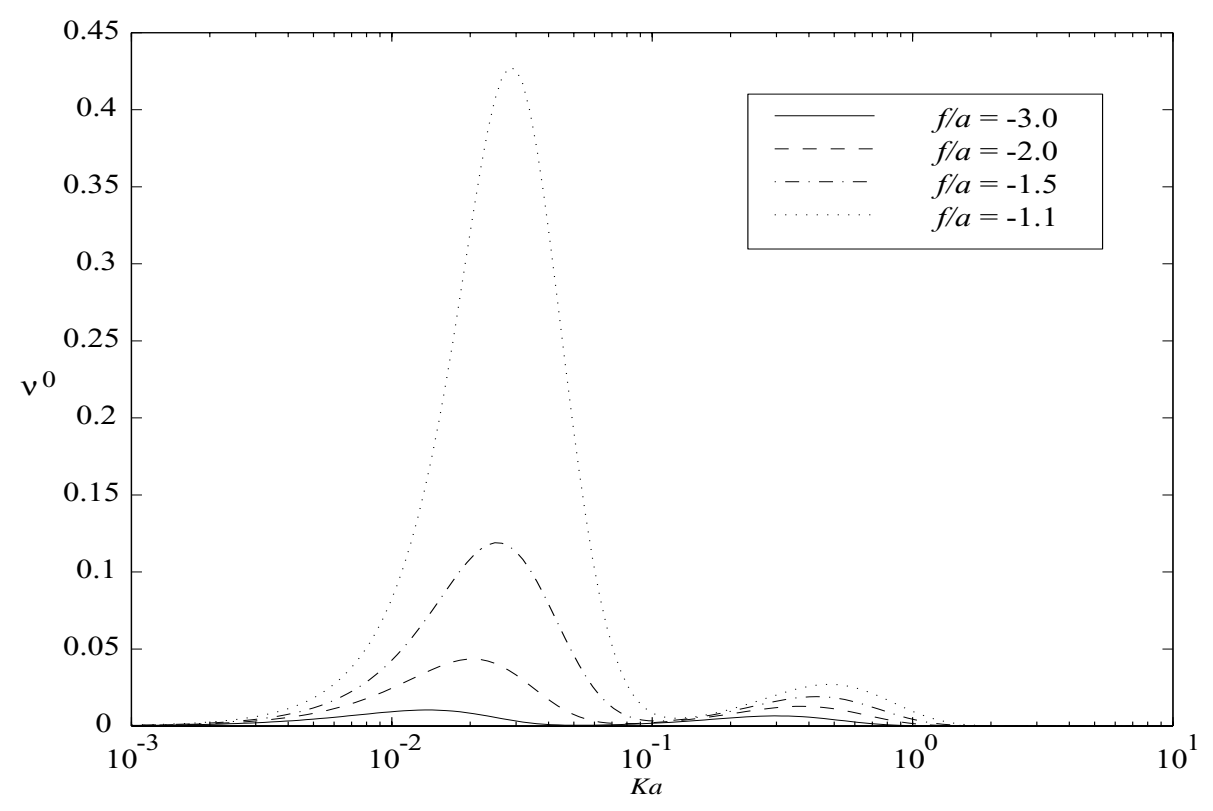

Figure 1: Damping coefficient $\nu^{0}$ (heave) plotted against $K a$ for a submerged sphere at different depths in the lower fluid layer; $\rho=0.95$ and $d / a=2.0$.

Figures 3 and 4 show the added-mass coefficients for heave and sway respectively. As the immersion depth increases the added-mass curves tend towards the constant value of $1 / 2$, which is the added-mass of a sphere oscillating in an infinite expanse of fluid. When the sphere is close to the interface, the deviation from $1 / 2$ is greater in the case of heave. It is again noticeable that there is an effect due to the presence of the interface near $k a=1$ and a much smaller effect due to the presence of the free surface near $K a=1$. In the limit as $K a \rightarrow 0$, the interface boundary conditions (2.3) and (2.4) reduce to $\phi_{z}^{I I}=0$ and we get the same results in the long-wave limit as if the interface were the free-surface in a single-layer fluid. Thus the solid curve in figure 2 of Linton (1991) tends to the same value as $K a \rightarrow 0$ as the $f / a=-1.5$ curve in figure 3 here.

\subsection{Sphere in upper fluid layer}

For problems involving a sphere in the upper fluid layer we need to develop multipoles singular at $z=f>0$. This can be done in much the same way as for the case $f<0$ and the details will be omitted. Suitable multipoles are

$$
\begin{aligned}
\phi_{n}^{I m} & =\left(\frac{a}{r}\right)^{n+1} P_{n}^{m}(\cos \theta)+\frac{(-1)^{m+n} a^{n+1}}{(n-m) !} f_{0}^{\infty} u^{n}\left[A_{U}(u) \mathrm{e}^{u z}+B_{U}(u) \mathrm{e}^{-u z}\right] J_{m}(u R) \mathrm{d} u \\
\phi_{n}^{I I m} & =\frac{(-1)^{m+n} a^{n+1}}{(n-m) !} f_{0}^{\infty} u^{n} J_{m}(u R) C_{U}(u) \mathrm{e}^{u z} \mathrm{~d} u,
\end{aligned}
$$

where

$$
\begin{aligned}
& A_{U}(u)=(u+K) \mathrm{e}^{-2 u d}\left[(-1)^{m+n+1}(u-K \sigma) \mathrm{e}^{u f}-(u-K) \mathrm{e}^{-u f}\right] /(u-K) h(u), \\
& B_{U}(u)=\left[(-1)^{m+n+1}(u+K) \mathrm{e}^{u(f-2 d)}-(u-K) \mathrm{e}^{-u f}\right] / h(u), \\
& C_{U}(u)=K(1-\sigma) B_{U}(u) /(u-K),
\end{aligned}
$$




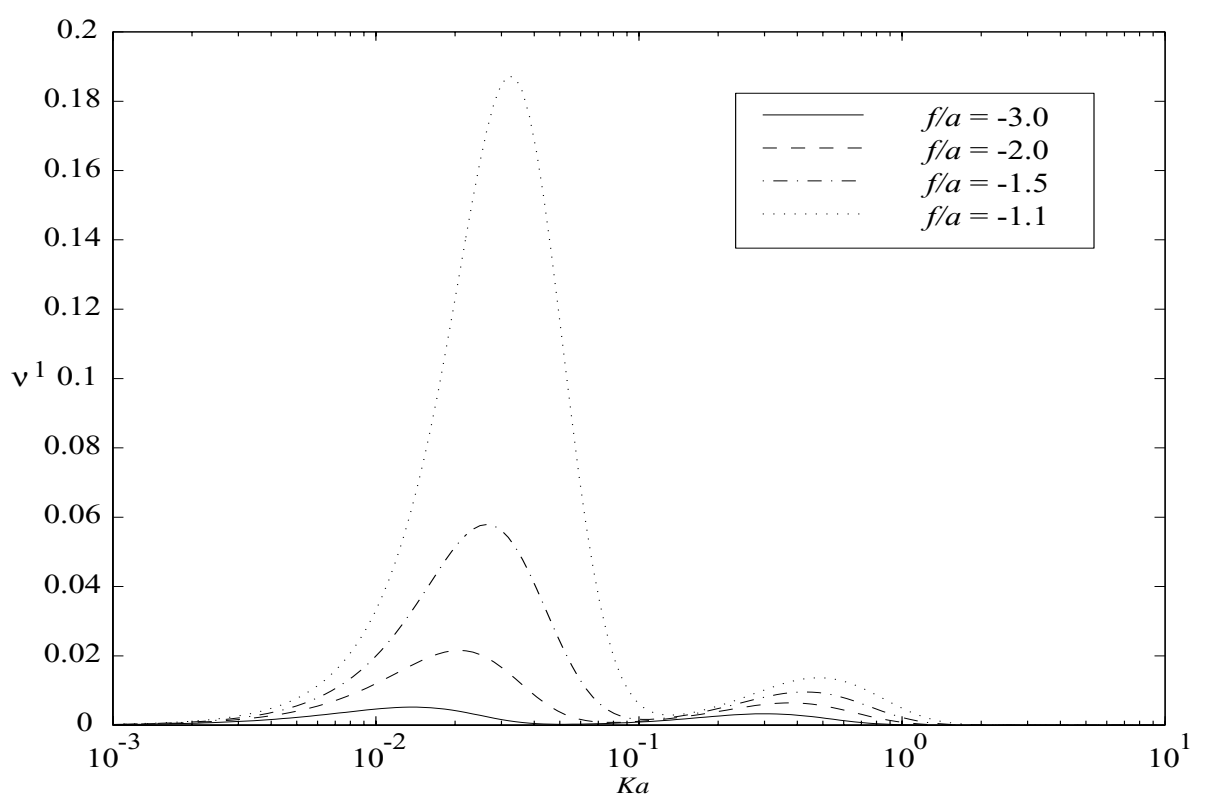

Figure 2: Damping coefficient $\nu^{1}$ (sway) plotted against $K a$ for a submerged sphere at different depths in the lower fluid layer; $\rho=0.95$ and $d / a=2.0$.

with $h(u)$ given by (3.8) as before. The multipoles are expanded about $r=0$ by using (3.12), giving

$$
\phi_{n}^{I m}=\left(\frac{a}{r}\right)^{n+1} P_{n}^{m}(\cos \theta)+\sum_{s=m}^{\infty}\left(\frac{r}{a}\right)^{s} B_{n s}^{m} P_{s}^{m}(\cos \theta)
$$

where

$$
B_{n s}^{m}=\frac{(-1)^{m+n} a}{(n-m) !(s+m) !} f_{0}^{\infty}(a u)^{n+s}\left[\mathrm{e}^{u f} A_{U}(u)+(-1)^{m+s} \mathrm{e}^{-u f} B_{U}(u)\right] \mathrm{d} u .
$$

The far field form of $\phi_{n}^{m}$, in the lower fluid, is given by

$$
\phi_{n}^{m} \sim \frac{(-1)^{n} \mathrm{i}^{m+1} a^{n+1}}{(n-m) !}\left(\frac{2 \pi}{R}\right)^{1 / 2}\left[K^{n-1 / 2} \mathrm{e}^{\mathrm{i} K R} C_{U}^{K} \mathrm{e}^{K z}+k^{n-1 / 2} \mathrm{e}^{\mathrm{i} k R} C_{U}^{k} \mathrm{e}^{k z}\right] \mathrm{e}^{-\mathrm{i} \pi / 4}
$$

as $K R \rightarrow \infty$, where

$$
\begin{aligned}
C_{U}^{K} & =\frac{(-1)^{m+n+1} 2 K(1-\sigma) \mathrm{e}^{K(f-2 d)}}{2 \mathrm{e}^{-2 u d}-1+\sigma} \\
C_{U}^{k} & =\frac{K(1-\sigma)\left[(-1)^{m+n+1}(K+k) \mathrm{e}^{k(f-2 d)}-(k-K) \mathrm{e}^{-k f}\right]}{(k-K)\left[(1-2 d(K+k)) \mathrm{e}^{-2 k d}-1\right]} .
\end{aligned}
$$

The velocity potential can be expanded in terms of these multipoles exactly as in (3.16) and then application of the body boundary condition leads to the infinite system of linear equations

$$
b_{s}^{m}-\frac{s}{s+1} \sum_{n=1}^{\infty} B_{n s}^{m} b_{n}^{m}=-\frac{\delta_{1 s}}{2} \quad s \geq 1 \quad m=0,1
$$




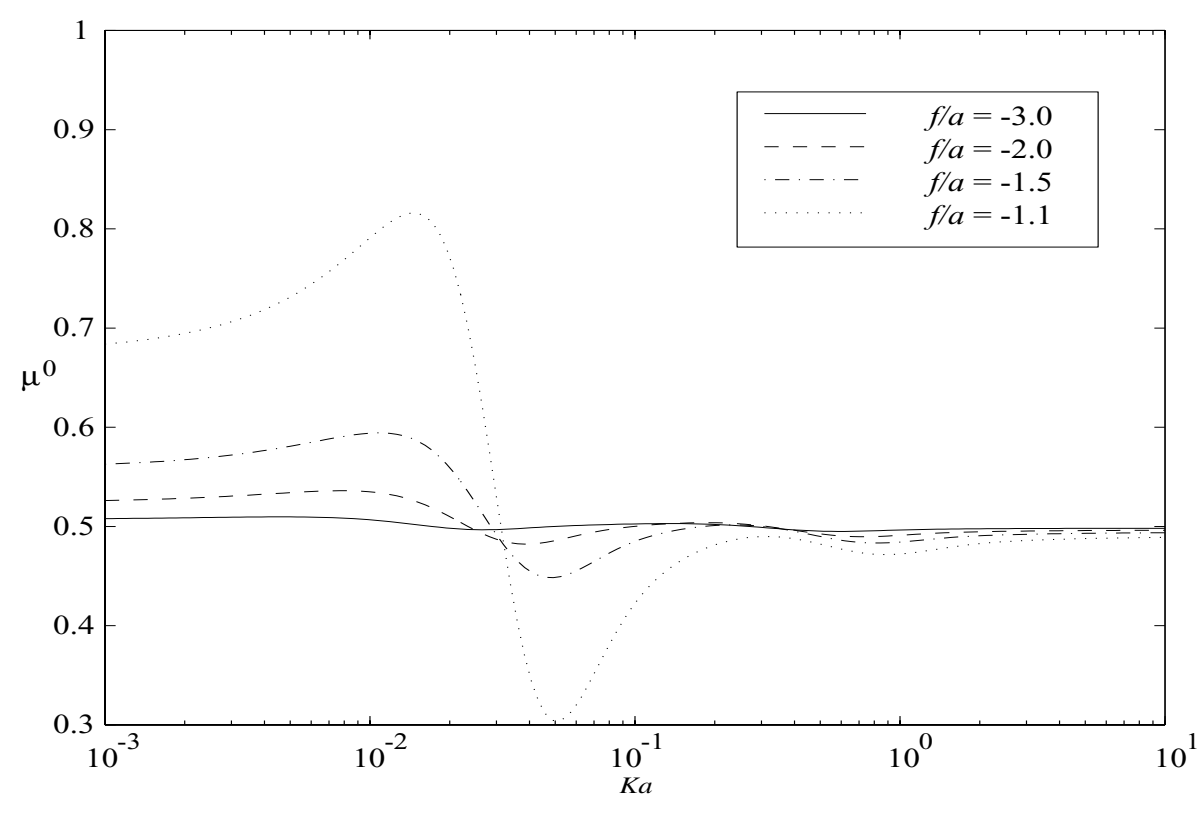

Figure 3: Added mass coefficient $\mu^{0}$ (heave) plotted against $K a$ for a submerged sphere at different depths in the lower fluid layer; $\rho=0.95$ and $d / a=2.0$.

for the unknown coefficients $b_{n}^{m}$. Once this system has been solved, the non-dimensional added mass and damping coefficients are given by (3.20) exactly as before. The identity relating the damping coefficient to the radiated energy, which serves as a numerical check on the results, is now

$$
\operatorname{Im}\left(b_{1}^{m}\right)=-\frac{\pi a}{4}\left(J_{K}\left|\sum_{n=1}^{\infty} \frac{(-K a)^{n} b_{n}^{m} C_{U}^{K}}{(n-m) !}\right|^{2}+J_{k}\left|\sum_{n=1}^{\infty} \frac{(-k a)^{n} b_{n}^{m} C_{U}^{k}}{(n-m) !}\right|^{2}\right)
$$

Note that the residues of $C_{U}$ are included in the summations because they depend on $n$.

Curves of added mass and damping coefficients for spheres in the upper fluid layer are shown in figures $5-8$. In all the curves $\rho$ is 0.95 and $d / a$ is 4.0 . Each plot shows the results obtained for four different submersion depths, $f / a$, of the sphere. The values of $f / a$ have been chosen so there are results for a sphere close to the interface $(f / a=1.1)$, close to the free surface $(f / a=2.9)$ and at two intermediate values $(f / a=1.7$ and 2.3).

Figures 5 and 6 show the damping coefficients for heave and sway motion respectively. The two cases lead to similar results, but those for heave motion are greater than those obtained for sway. As with the results for the sphere in the lower region there are two local maximums, one near $k a=1$ which corresponds to waves being generated on the interface and one near $K a=1$ which corresponds to waves on the free surface. When the sphere is close to the interface $(f / a=1.1)$ the first local maximum is greater as more waves are generated on the interface than on the free surface. As the sphere approaches the free surface the ability to make waves on the interface decreases whilst it becomes easier to generate waves on the free surface; thus the first maximum decreases and the second increases. The maximum value of the damping coefficient is greater when $f / a=2.9$ (when the distance from the free surface is $0.1 a$ ) than for the case $f / a=1.1$ (when the distance from the interface is $0.1 a$ ) showing that the ability to make waves on the free-surface is greater than that on the interface. It is also noteworthy that the range of values of $K a$ 


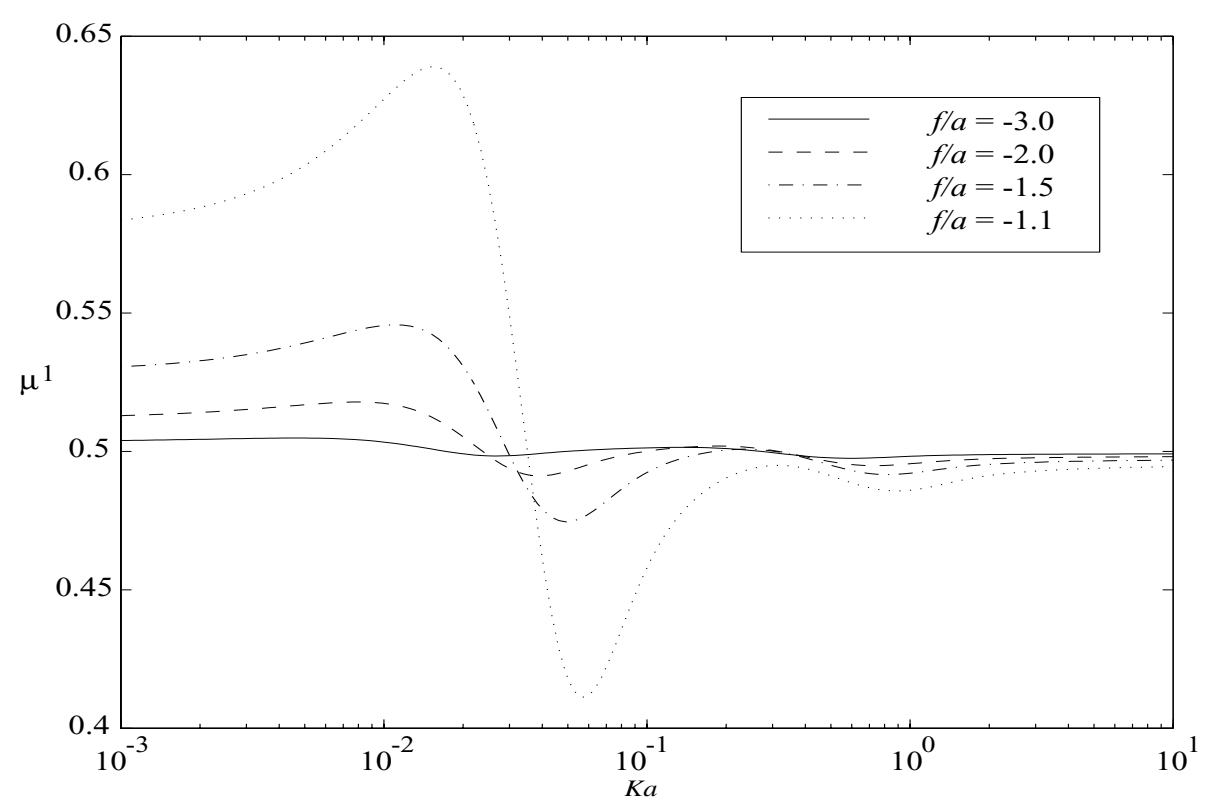

Figure 4: Added mass coefficient $\mu^{1}$ (sway) plotted against $K a$ for a submerged sphere at different depths in the lower fluid layer; $\rho=0.95$ and $d / a=2.0$.

over which there is a noticeable effect on the free surface is an order of magnitude bigger than the range for which the interface is affected.

The added-mass coefficients for heave and sway are shown in figures 7 and 8 respectively and again the deviations from the infinite fluid value of $1 / 2$ are greater for heave motion than for sway motion. The effect of the interface is pronounced when $f / a=1.1$ as can be seen by the fact that large variations occur in the added-mass around $k a=1$ ( $K a \approx 0.025)$ whereas for $f / a=2.9$ it is the free-surface effect which dominates with large variations around $K a=1$. In the limit as $K a \rightarrow 0$ the problem reduces to that of a sphere oscillating between parallel planes and so we get the same limiting value for $f / a=1.1$ and 2.9 (as in each case the sphere is a distance $0.1 a$ from one of the walls) and similarly the same limiting value is obtained for $f / a=1.7$ and 2.3 (in each case the sphere is $0.7 a$ from one of the walls). Though not present on these figures, negative added-mass can occur for the heave problem when the sphere is either very close to the free surface or to the interface. This phenonmenon, which does not appear to occur for sway, or when the sphere is below the interface is discussed by McIver and Evans (1984).

\section{Scattering problems}

In this section we will solve the problem of the scattering of an incident plane wave, of wavenumber either $K$ or $k$, by a submerged sphere, first situated in the lower layer and then above the interface. In each case the total scattering potential can be decomposed into two parts:

$$
\phi=\phi_{\mathrm{inc}}+\phi_{S}
$$

where $\phi_{\text {inc }}$ is the potential representing the incident plane wave (given, up to an arbitrary multiplicative constant by (2.15) if the incident wave has wavenumber $K$ and by (2.13) and 


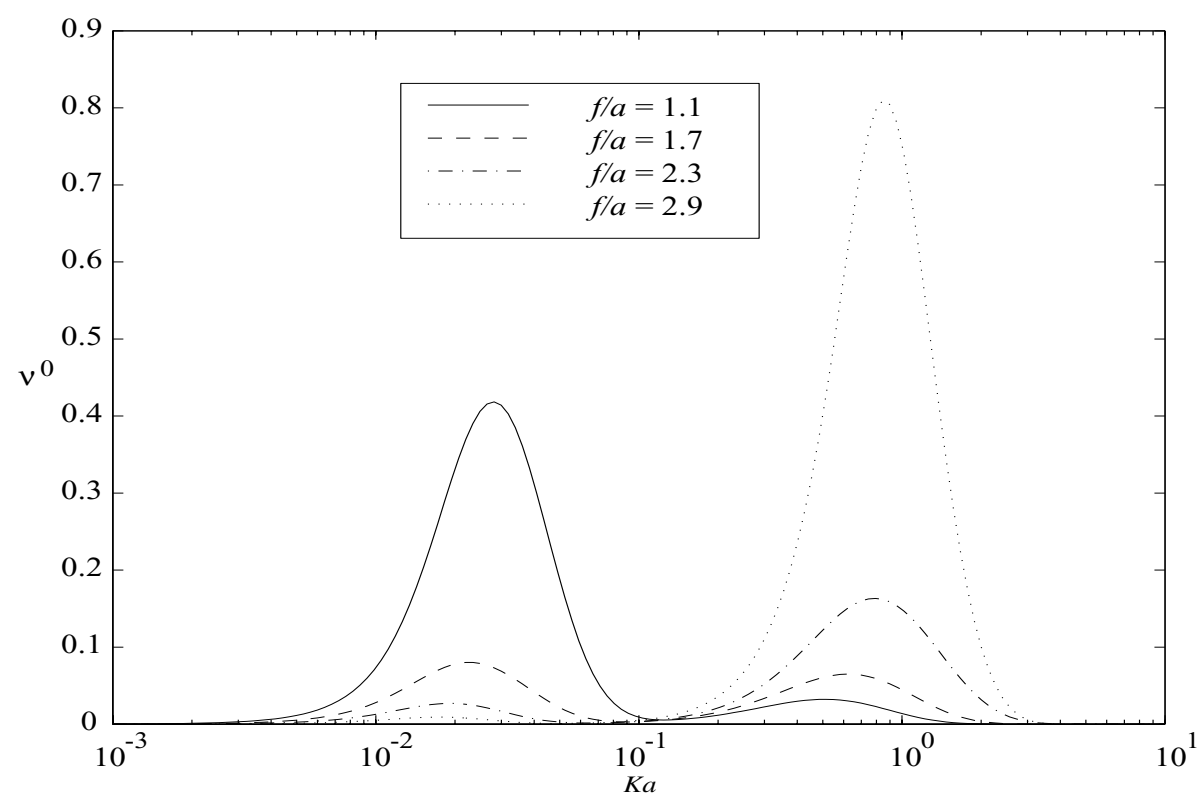

Figure 5: Damping coefficient $\nu^{0}$ (heave) plotted against $K a$ for a submerged sphere at different depths in the upper fluid layer; $\rho=0.95$ and $d / a=4.0$.

(2.14) if the incident wave has wavenumber $k$ ) and $\phi_{S}$ therefore must satisfy $(2.2)-(2.5)$, the body boundary condition

$$
\frac{\partial \phi_{S}}{\partial r}=-\frac{\partial \phi_{\mathrm{inc}}}{\partial r} \quad \text { on } r=a
$$

and behave as an outgoing cylindrical wave far from the sphere. Without loss of generality we can assume that the incident wave is from $x=-\infty$ so that $\alpha_{\mathrm{inc}}=0$.

\subsection{Sphere in lower fluid layer}

First we consider an incident plane wave of wavenumber $K$ and amplitude $A$ on the free surface $(z=d)$ whose potential can be expanded in spherical polar coordinates using Abramowitz and Stegun (1965), eqns (9.1.44), (9.1.45), and (3.12) above, to give

$$
\begin{aligned}
\phi_{\text {inc }} & =-\frac{\mathrm{i} g A}{\omega} \mathrm{e}^{K(z-d)} \mathrm{e}^{\mathrm{i} K R \cos \alpha} \\
& =-\frac{\mathrm{i} g A}{\omega} \mathrm{e}^{K(f-d)} \sum_{m=0}^{\infty} \epsilon_{m} \mathrm{i}^{m} \cos m \alpha \sum_{s=m}^{\infty} \frac{(K r)^{s}}{(s+m) !} P_{s}^{m}(\cos \theta),
\end{aligned}
$$

where $\epsilon_{0}=1, \epsilon_{m}=2$ for $m \geq 1$.

For the radiation problems considered in the previous section the dependence on the azimuthal angle $\alpha$ was known, but here it is not (apart from the fact that it is even) and so we must use a more general multipole expansion. We write

$$
\phi_{S}=-\frac{\mathrm{i} g A}{\omega} \sum_{m=0}^{\infty} \sum_{n=m_{1}}^{\infty} c_{n}^{m} \phi_{n}^{m} \cos m \alpha
$$

where $m_{1}=\max (m, 1)$ and $\phi_{n}^{m}$ is given (in the lower fluid layer) by (3.13). If we then apply the boundary condition (4.2) and use the orthogonality of the associated Legendre 


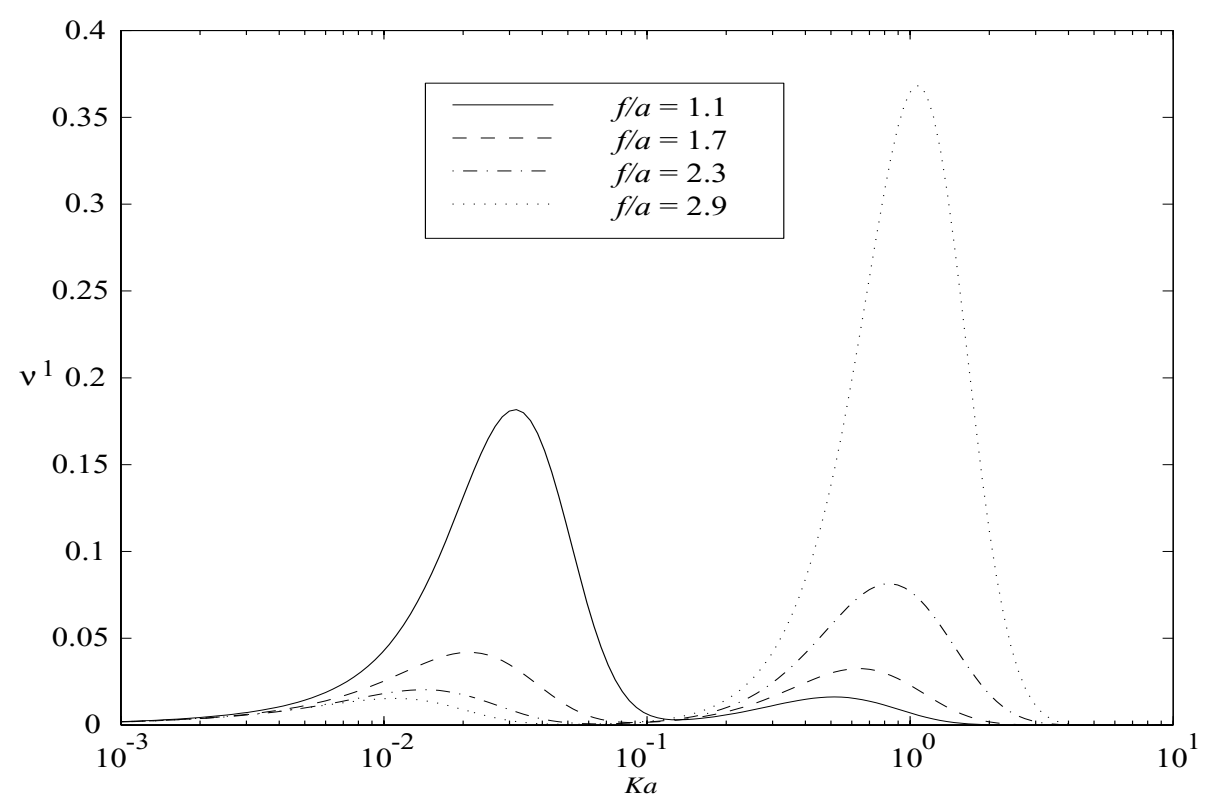

Figure 6: Damping coefficient $\nu^{1}$ (sway) plotted against $K a$ for a submerged sphere at different depths in the upper fluid layer; $\rho=0.95$ and $d / a=4.0$.

functions and the functions $\cos m \alpha$ we can derive an infinite system of equations for the sets of coefficients $c_{n}^{m}, n \geq m_{1}$ for each $m \geq 0$, which is

$$
c_{s}^{m}-\frac{s}{s+1} \sum_{n=m_{1}}^{\infty} A_{n s}^{m} c_{n}^{m}=\frac{\epsilon_{m} \mathrm{i}^{m} s(K a)^{s} \mathrm{e}^{K(f-d)}}{(s+1)(s+m) !}, \quad s \geq m_{1} .
$$

These systems can be solved by truncation as before, but now there is an additional truncation parameter, namely the number of systems that are solved. In the computations presented below two $4 \times 4$ systems were solved.

The vertical and horizontal exciting forces on the sphere, $f_{K}^{0}$ and $f_{K}^{1}$, can be calculated from (2.30). We obtain

$$
f_{K}^{0}=-\frac{4}{3} \pi a^{2} \rho^{I I} g A\left(K a \mathrm{e}^{K(f-d)}+c_{1}^{0}+\sum_{n=1}^{\infty} A_{n 1}^{0} c_{n}^{0}\right)
$$

and

$$
f_{K}^{1}=-\frac{4}{3} \pi a^{2} \rho^{I I} g A\left(\mathrm{i} K a \mathrm{e}^{K(f-d)}+c_{1}^{1}+\sum_{n=1}^{\infty} A_{n 1}^{1} c_{n}^{1}\right) .
$$

These can be simplified using (4.6) with $s=1$, giving

$$
\overline{f_{K}^{0}} \equiv\left|\frac{f_{K}^{0}}{a^{2} \rho^{I I} g A}\right|=4 \pi\left|c_{1}^{0}\right|, \quad \overline{f_{K}^{1}} \equiv\left|\frac{f_{K}^{1}}{a^{2} \rho^{I I} g A}\right|=4 \pi\left|c_{1}^{1}\right| .
$$

The vertical and horizontal exciting forces are related to the heave and sway radiation problems through (2.31). We find that

$$
\begin{aligned}
c_{1}^{0} & =-\frac{J_{K}}{2} \mathrm{e}^{-K d} C_{L}^{K} \sum_{n=1}^{\infty} \frac{b_{n}^{0}(K a)^{n}}{n !}, \\
c_{1}^{1} & =-\frac{\mathrm{i} J_{K}}{2} \mathrm{e}^{-K d} C_{L}^{K} \sum_{n=1}^{\infty} \frac{b_{n}^{1}(K a)^{n}}{(n-1) !},
\end{aligned}
$$




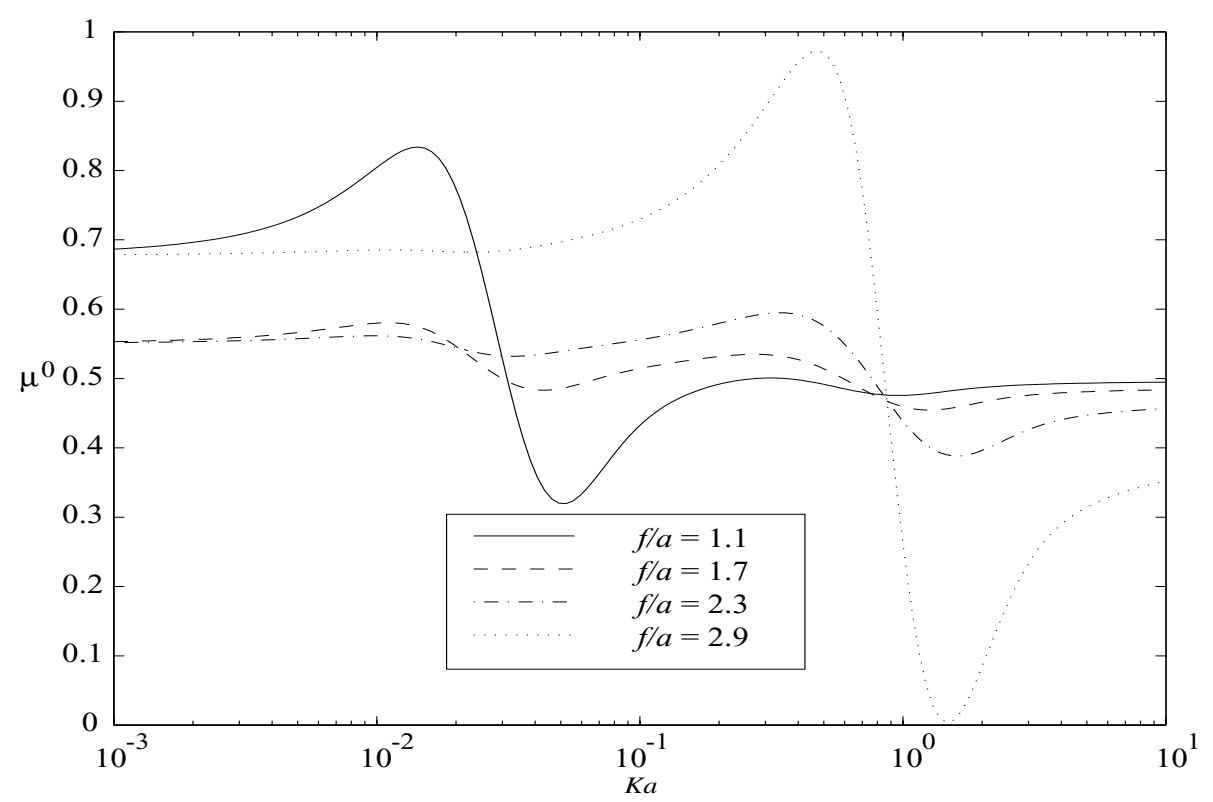

Figure 7: Added mass coefficient $\mu^{0}$ (heave) plotted against $K a$ for a submerged sphere at different depths in the upper fluid layer; $\rho=0.95$ and $d / a=4.0$.

where the coefficients $b_{n}^{m}$ are the solutions of (3.17). These identities were used as a numerical check on the results obtained from the radiation and scattering problems.

Next we consider the case of an incident plane wave of amplitude $A$ on the interface $(z=0)$ and wavenumber $k$, described by

$$
\phi_{\text {inc }}^{I}=-\frac{\mathrm{i} g A K}{\omega k} g(z) \mathrm{e}^{\mathrm{i} k R \cos \alpha}, \quad \phi_{\text {inc }}^{I I}=-\frac{\mathrm{i} g A K}{\omega k} \mathrm{e}^{k z} \mathrm{e}^{\mathrm{i} k R \cos \alpha} .
$$

The analysis is very similar to that given above for an incident wave of wavenumber $K$. We use the same expansion for $\phi_{S}$ as before, equation (4.5), but denote the unknown coefficients by $d_{n}^{m}$ and we obtain the infinite system of equations

$$
d_{s}^{m}-\frac{s}{s+1} \sum_{n=m_{1}}^{\infty} A_{n s}^{m} d_{n}^{m}=\frac{\epsilon_{m} \mathrm{i}^{m} s K a(k a)^{s-1} \mathrm{e}^{k f}}{(s+1)(s+m) !}, \quad s \geq m_{1},
$$

for each $m \geq 0$.

The expressions for the vertical and horizontal exciting forces are

$$
\overline{f_{k}^{0}} \equiv\left|\frac{f_{k}^{0}}{a^{2} \rho^{I I} g A}\right|=4 \pi\left|d_{1}^{0}\right|, \quad \overline{f_{k}^{1}} \equiv\left|\frac{f_{k}^{1}}{a^{2} \rho^{I I} g A}\right|=4 \pi\left|d_{1}^{1}\right|
$$

and the formulas connecting this scattering problem to the heave and sway radiation problems are now, from (2.32),

$$
\begin{aligned}
& d_{1}^{0}=-\frac{J_{k}}{2} C_{L}^{k} \sum_{n=1}^{\infty} \frac{b_{n}^{0} K a(k a)^{n-1}}{n !}, \\
& d_{1}^{1}=-\frac{\mathrm{i} J_{k}}{2} C_{L}^{k} \sum_{n=1}^{\infty} \frac{b_{n}^{1} K a(k a)^{n-1}}{(n-1) !},
\end{aligned}
$$




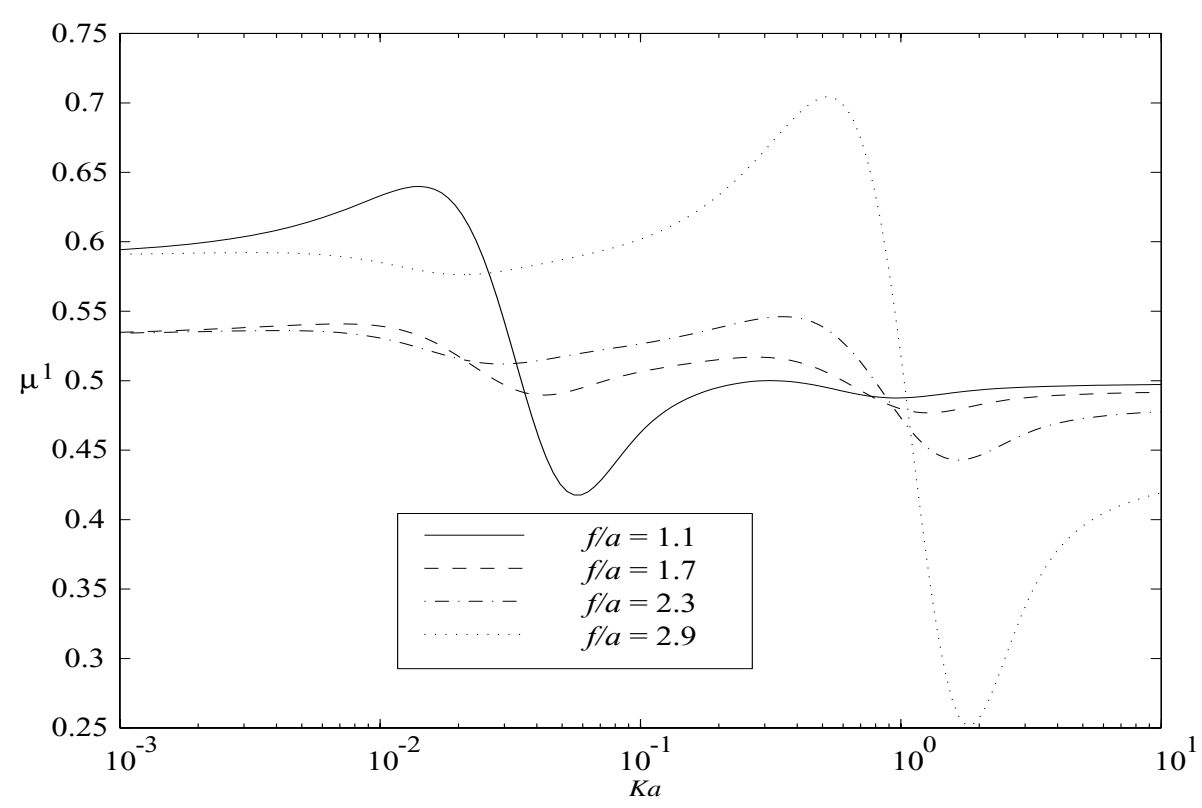

Figure 8: Added mass coefficient $\mu^{1}$ (sway) plotted against $K a$ for a submerged sphere at different depths in the upper fluid layer; $\rho=0.95$ and $d / a=4.0$.

where the coefficients $b_{n}^{m}$ are the solutions of (3.17).

Equations (3.20), (4.9)-(4.11), (4.14)-(4.16) can be combined with (3.24) with $m=0$ and 1 to give

$$
\nu^{m}=\frac{3 a}{16 \pi}\left(\frac{\mathrm{e}^{2 K d}}{J_{K}} \bar{f}_{K}^{m}+\frac{k^{2}}{K^{2} J_{k}} \bar{f}_{k}^{m}\right)
$$

which relates the heave and sway damping coefficients to the vertical and horizontal exciting forces respectively.

Figures 9-12 show curves of $\overline{f^{0}}$ and $\overline{f^{1}}$ plotted against $K a$ for both scattering problems considered above. In each figure there are four curves corresponding to different immersion depths of the sphere in the lower region. These immersion depths, $f / a=-1.1,-1.5$, -2 and -3 are the same as those used in the section 3.1, as are the values $\rho=0.95$ and $d / a=2.0$. Figures 9 and 10 show, respectively, the non-dimensionalized vertical and horizontal exciting forces on the sphere due to an incident wave of wavenumber $K$. The two sets of curves are very similar and show that, as one would expect, the forces increase the closer the sphere is to the interface (and hence to the free surface).

Figures 11 and 12 show curves for the case of an incident wave of wavenumber $k$. Again, the figures for the vertical and horizontal forces look similar but the results obtained for $\overline{f_{k}^{0}}$ are greater that those for $\overline{f_{k}^{1}}$. The exciting forces increase as the surface of the sphere approaches the interface. The incident wave in figures 9 and 10 is associated with the free surface, but here it is associated with the interface which is much closer to the sphere; as a result the forces are rather larger than those in the previous case. Also, the maximum forces occur for much longer waves than for an incident wave of wavenumber $K$. 


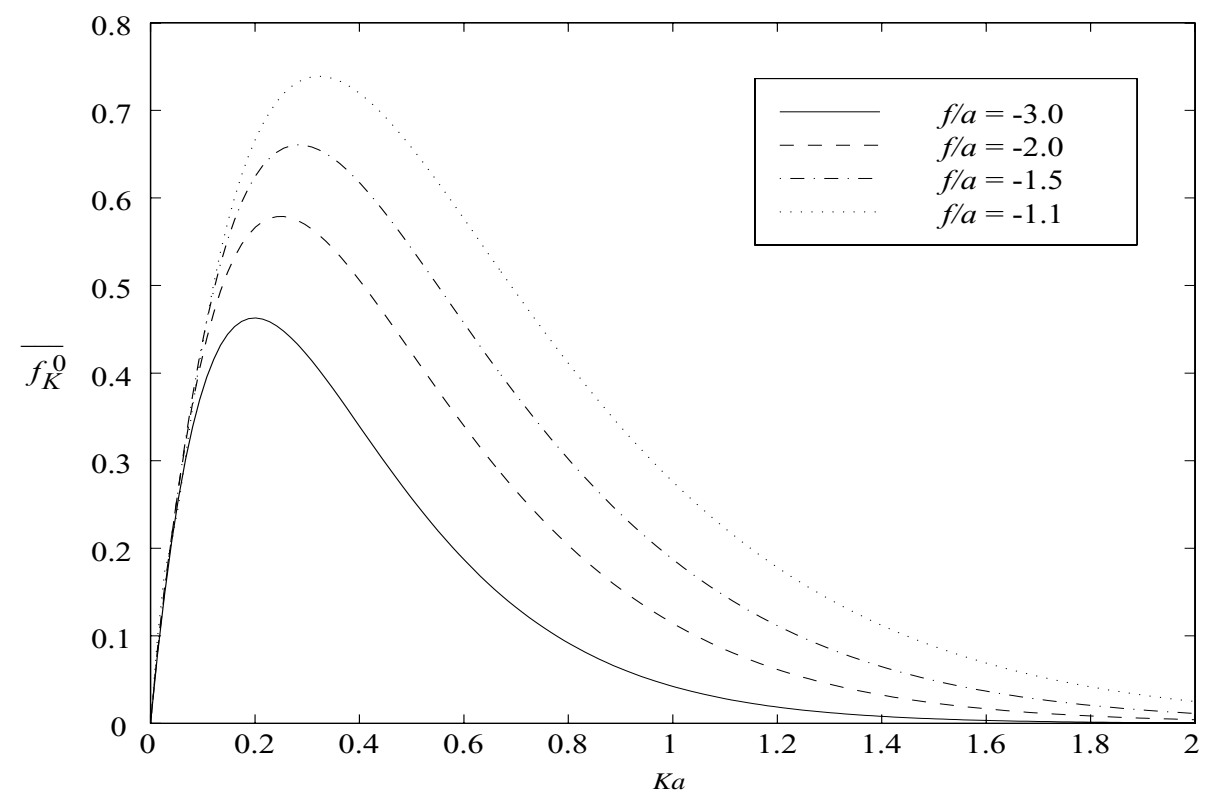

Figure 9: Non-dimensionalized vertical exciting force $\overline{f_{K}^{0}}$ plotted against $K a$ for different submersion depths in the lower fluid layer; $\rho=0.95$ and $d / a=2.0$.

\subsection{Sphere in upper fluid layer}

An incident plane wave of wavenumber $K$ and amplitude $A$ on the free surface $(z=d)$ has the same form in the upper layer as in the lower layer, given by (4.4). The potential $\phi_{S}$ can again be expanded using (4.5), but we now use the multipole expansions developed for the upper fluid layer, (3.30). The details of the solution procedure will be omitted. For each $m \geq 0$ the coefficients $c_{n}^{m}$ satisfy the infinite system of equations

$$
c_{s}^{m}-\frac{s}{s+1} \sum_{n=m_{1}}^{\infty} B_{n s}^{m} c_{n}^{m}=\frac{\epsilon_{m} \mathrm{i}^{m} s(K a)^{s} \mathrm{e}^{K(f-d)}}{(s+1)(s+m) !}, \quad s \geq m_{1} .
$$

and the non-dimensional vertical and horizontal exciting forces on the sphere are given by

$$
\overline{f_{K}^{0}} \equiv\left|\frac{f_{K}^{0}}{a^{2} \rho^{I} g A}\right|=4 \pi\left|c_{1}^{0}\right|, \quad \overline{f_{K}^{1}} \equiv\left|\frac{f_{K}^{1}}{a^{2} \rho^{I} g A}\right|=4 \pi\left|c_{1}^{1}\right| .
$$

The solution is related to the heave and sway radiation problems for a sphere in the upper fluid through the equations

$$
\begin{aligned}
& c_{1}^{0}=-\frac{J_{K}}{2 \rho} \mathrm{e}^{-K d} \sum_{n=1}^{\infty} \frac{b_{n}^{0} C_{U}^{K}}{n !}(-K a)^{n}, \\
& c_{1}^{1}=\frac{\mathrm{i} J_{K}}{2 \rho} \mathrm{e}^{-K d} \sum_{n=1}^{\infty} \frac{b_{n}^{1} C_{U}^{K}}{(n-1) !}(-K a)^{n},
\end{aligned}
$$

where the coefficients $b_{n}^{m}$ are the solutions of (3.35).

For the case of an incident wave of wavenumber $k$ (and amplitude $A$ on the interface $z=0$ ) we note that in region $I$ this takes the form

$$
\phi_{\text {inc }}^{I}=-\frac{\mathrm{i} g A K}{\omega k} g(z) \mathrm{e}^{\mathrm{i} k R \cos \alpha}=-\frac{\mathrm{i} g A K}{\omega k}\left[\frac{K \sigma-k}{K(\sigma-1)} \mathrm{e}^{k z}+\frac{K-k}{K(\sigma-1)} \mathrm{e}^{-k z}\right] \mathrm{e}^{\mathrm{i} k R \cos \alpha},
$$




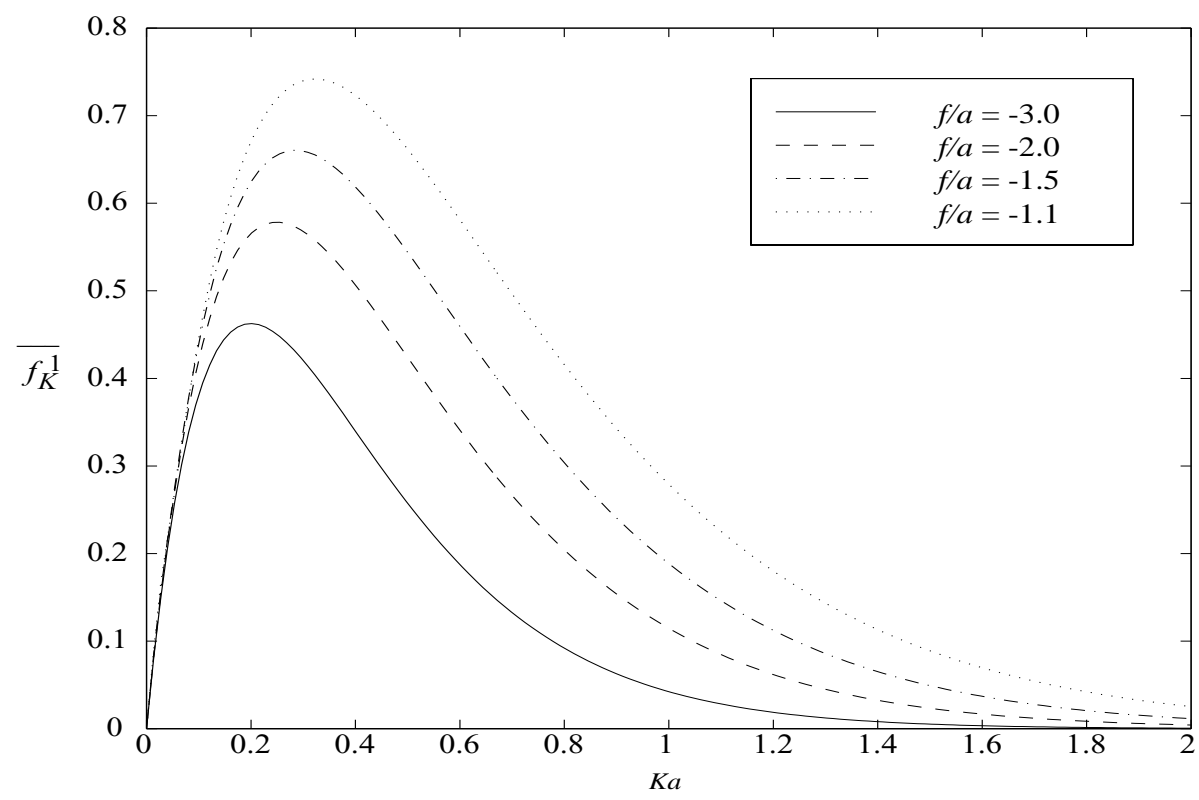

Figure 10: Non-dimensionalized horizontal exciting force $\overline{f_{K}^{1}}$ plotted against $K a$ for different submersion depths in the lower fluid layer; $\rho=0.95$ and $d / a=2.0$.

which can be expanded in spherical polar coordinates, giving

$$
\begin{aligned}
\phi_{\mathrm{inc}}=-\frac{\mathrm{i} g A}{\omega k(\sigma-1)} & \sum_{m=0}^{\infty} \epsilon_{m} \mathrm{i}^{m} \cos m \alpha \\
& \times \sum_{s=m}^{\infty}\left[(K \sigma-k) \mathrm{e}^{k f}+(-1)^{m+s}(K-k) \mathrm{e}^{-k f}\right] \frac{(k r)^{s}}{(s+m) !} P_{s}^{m}(\cos \theta) .
\end{aligned}
$$

For each $m \geq 0$ the coefficients $d_{n}^{m}$ in the expansion for $\phi_{S}$ satisfy the infinite system of equations

$$
\begin{aligned}
d_{s}^{m}-\frac{s}{s+1} & \sum_{n=m_{1}}^{\infty} B_{n s}^{m} d_{n}^{m} \\
& =\frac{\epsilon_{m} \mathrm{i}^{m} s(k a)^{s}}{(s+1)(s+m) !} \frac{(K \sigma-k) \mathrm{e}^{k f}+(-1)^{m+s}(K-k) \mathrm{e}^{-k f}}{k(\sigma-1)}, \quad s \geq m_{1},
\end{aligned}
$$

and the non-dimensional vertical and horizontal exciting forces on the sphere are given by

$$
\overline{f_{k}^{0}} \equiv\left|\frac{f_{k}^{0}}{a^{2} \rho^{I} g A}\right|=4 \pi\left|d_{1}^{0}\right|, \quad \overline{f_{k}^{1}} \equiv\left|\frac{f_{k}^{1}}{a^{2} \rho^{I} g A}\right|=4 \pi\left|d_{1}^{1}\right| .
$$

We also have the relations

$$
\begin{aligned}
& d_{1}^{0}=-\frac{K J_{k}}{2 \rho k} \sum_{n=1}^{\infty} \frac{b_{n}^{0} C_{U}^{k}}{n !}(-k a)^{n} \\
& d_{1}^{1}=\frac{\mathrm{i} K J_{k}}{2 \rho k} \sum_{n=1}^{\infty} \frac{b_{n}^{1} C_{U}^{k}}{(n-1) !}(-k a)^{n}
\end{aligned}
$$




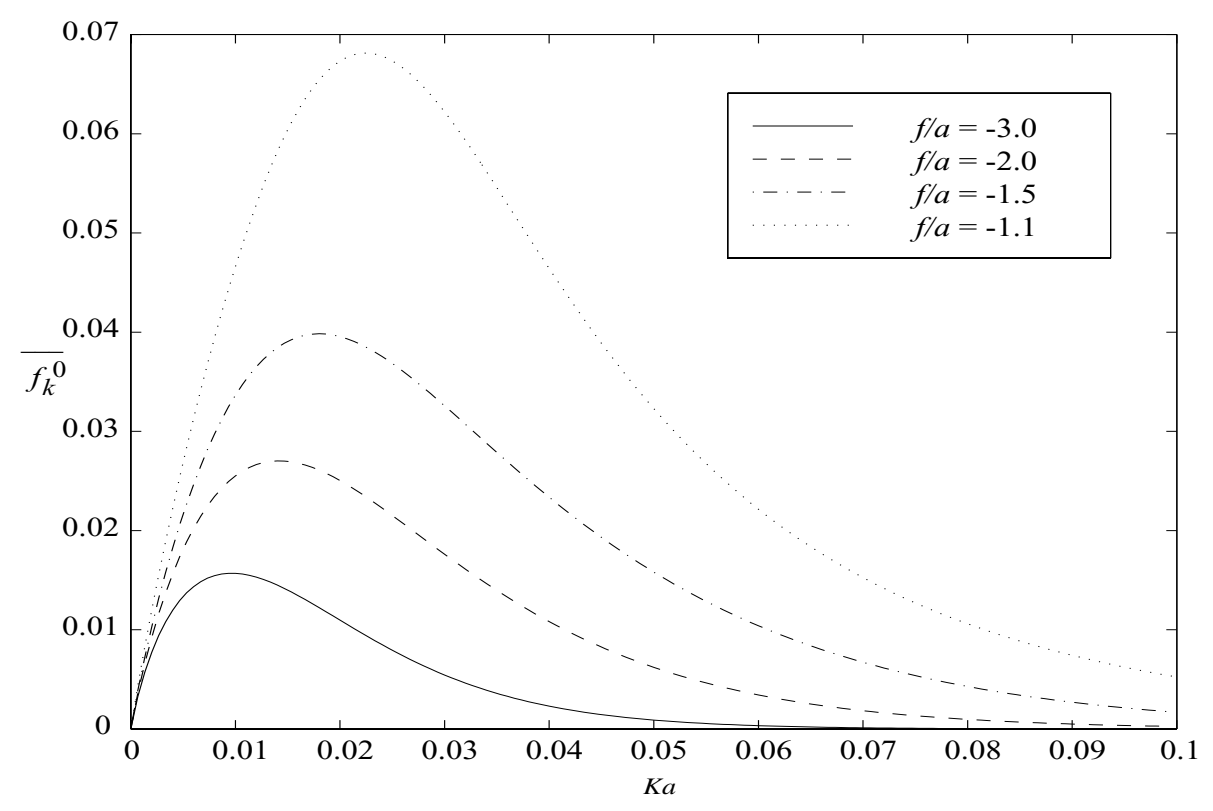

Figure 11: Non-dimensionalized vertical exciting force $\overline{f_{k}^{0}}$ plotted against $K a$ for different submersion depths in the lower fluid layer; $\rho=0.95$ and $d / a=2.0$.

where the coefficients $b_{n}^{m}$ are the solutions of (3.35), and

$$
\nu^{m}=\frac{3 a \rho^{2}}{16 \pi}\left(\frac{\mathrm{e}^{2 K d}}{J_{K}}{\overline{f_{K}^{m}}}^{2}+\frac{k^{2}}{K^{2} J_{k}}{\overline{f_{k}^{m}}}^{2}\right) .
$$

Figures 13-16 show curves of $\overline{f^{0}}$ and $\overline{f^{1}}$ plotted against $K a$ for incident wavenumbers $K$ and $k$ when $\rho=0.95$ and $d / a=4.0$. For each figure there are four curves corresponding to the immersion depths $f / a=1.1,1.7,2.3$ and 2.9. These immersion depths are the same as those used in section 3.2 Figures 13 and 14 show, respectively, the non-dimensionalized vertical and horizontal exciting forces on the sphere due to an incident wave of wavenumber $K$. The values obtained for $\overline{f_{K}^{0}}$ are larger than those for $\overline{f_{K}^{1}}$ and the curves are very similar (apart from the $K a$ values) to those shown in figures 11 and 12 in which the incident wave is associated with the interface and the sphere is in the lower layer. As one would expect, the forces are greater the closer the sphere is to the free surface. Figures 15 and 16 show curves for the case of an incident wave of wavenumber $k$. Again, the values obtained for the vertical forces are greater than those for the horizontal force but now the forces increase as the the sphere approaches the interface.

\section{Conclusion}

In this paper we have examined the relationships that exist between the solutions to three-dimensional radiation and scattering problems in two-layer fluids where the upper fluid is bounded above by a free surface and the lower (denser) fluid is infinite in extent. In such a situation propagating waves can exist at two different wavenumbers for any given frequency. A systematic derivation, using Green's theorem, of all the reciprocity relations for such problems has been carried out including extensions to the two-fluid case of the Haskind and Bessho-Newman relations. We have then used multipole expansions 


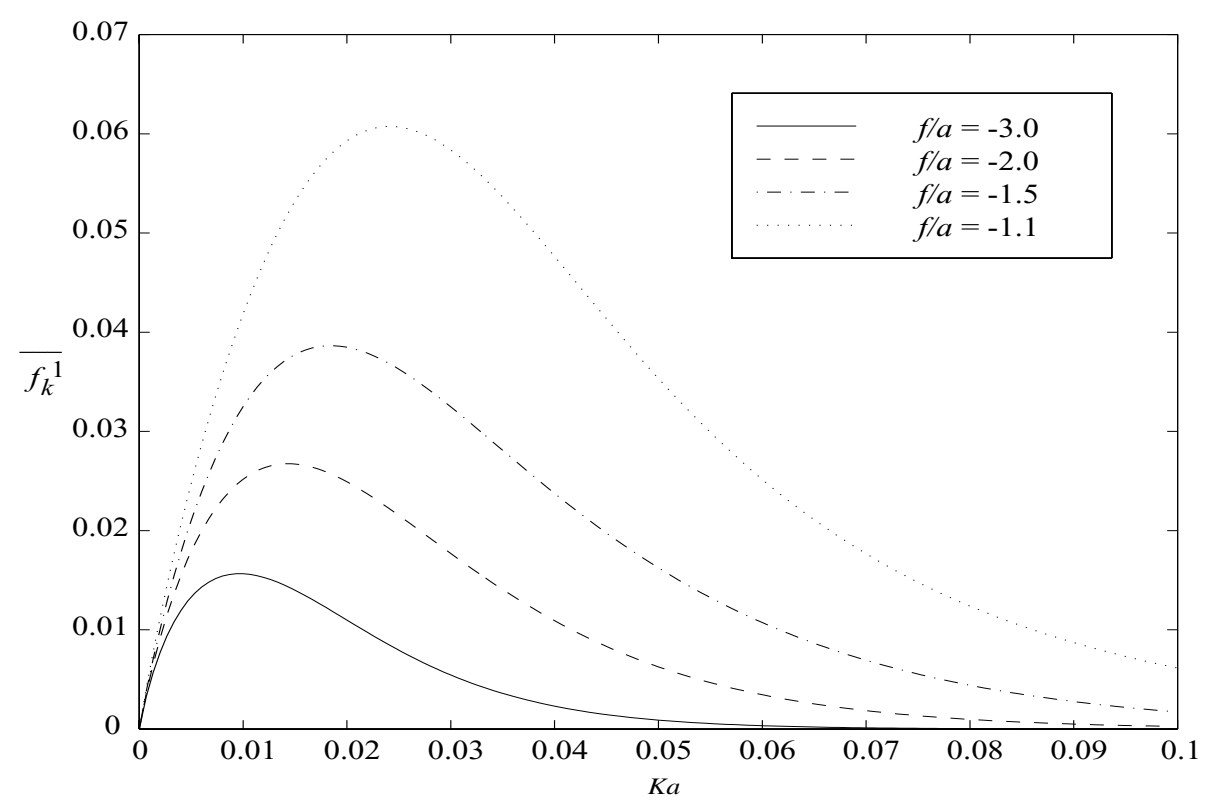

Figure 12: Non-dimensionalized horizontal exciting force $\overline{f_{k}^{1}}$ plotted against $K a$ for different submersion depths in the lower fluid layer; $\rho=0.95$ and $d / a=2.0$.

to solve radiation and scattering problems for a sphere situated entirely within either the upper or lower fluid layer.

One of the approximations that has been made is to model the interface between the two layers as being sharp, with a discontinuous density variation. A possible extension to the work described above would be to model the interface as a layer of finite thickness in which the density varies very rapidly. Such a layer is called a pycnocline. Some work in this direction using multipoles has been carried out by Gavrilov, Ermanyuk, and Sturova (1999) for the two-dimensional case of a two layer fluid which is bounded above and below by rigid walls and contains a horizontal circular cylinder.

\section{References}

Abramowitz, M., \& Stegun, I. A. (1965). Handbook of Mathematical Functions. Dover, New York.

Gavrilov, N., Ermanyuk, E., \& Sturova, I. (1999). Scattering of internal waves by a circular cylinder submerged in a stratified fluid. In Proc. 22nd Symposium on Naval Hydrodynamics, ONR.

Iooss, G. (1999). Gravity and capillary-gravity periodic travelling waves for two superposed fluid layers, one being of infinite depth. J. Math. Fluid Mech., 1, 24-61.

Lamb, H. (1932). Hydrodynamics (6th ed.). Cambridge University Press. Reprinted 1993.

Linton, C. M. (1991). Radiation and diffraction of water waves by a submerged sphere in finite depth. Ocean Engng., 18(1/2), 61-74.

Linton, C. M., \& Evans, D. V. (1992). The radiation and scattering of surface waves by a vertical circular cylinder in a channel. Phil. Trans. R. Soc. Lond., A, 338, 325357. 


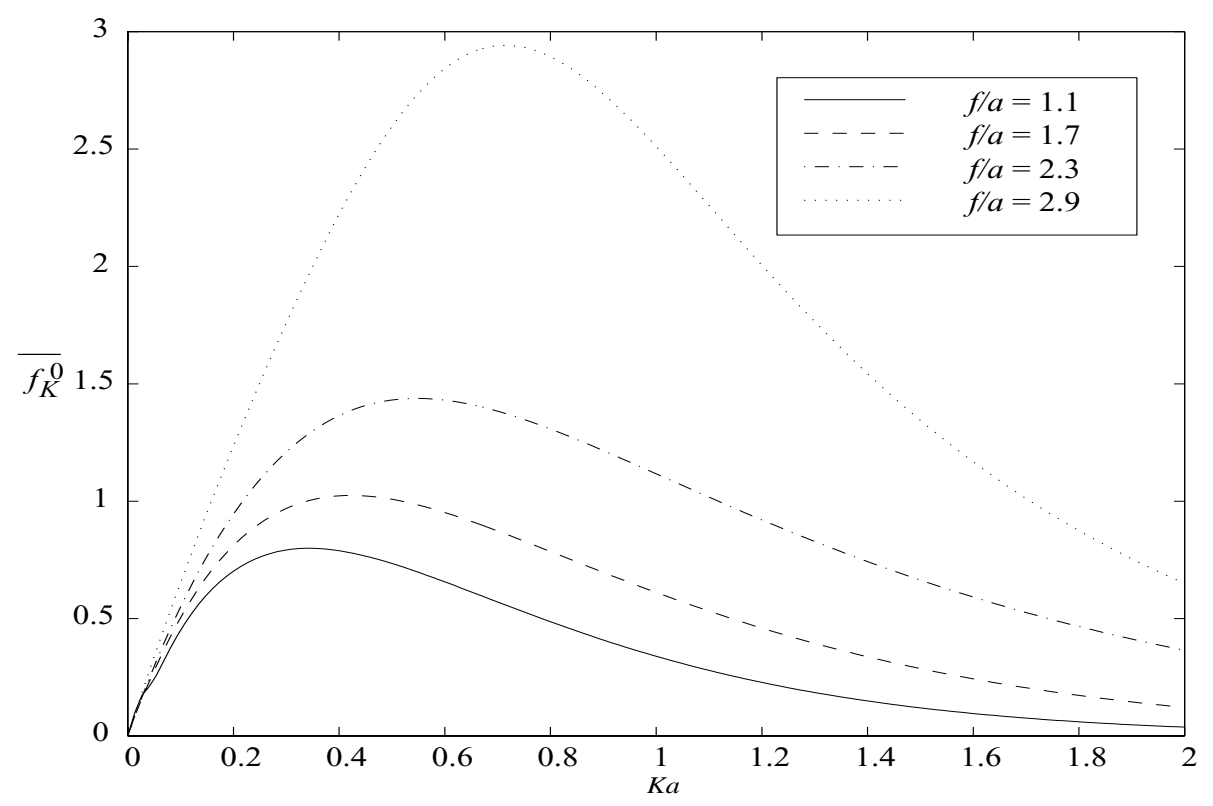

Figure 13: Non-dimensionalized vertical exciting force $\overline{f_{K}^{0}}$ due to an incident wave of wavenumber $K$, plotted against $K a$ for different submersion depths in the upper fluid layer; $\rho=0.95$ and $d / a=4.0$.

Linton, C. M., \& McIver, M. (1995). The interaction of waves with horizontal cylinders in two-layer fluids. J. Fluid Mech., 304, 213-229.

McIver, P., \& Evans, D. V. (1984). The occurrence of negative added mass in freesurface problems involving submerged oscillating bodies. J. Engng. Math., 18, 7-22.

MeI, C. C. (1983). The Applied Dynamics of Ocean Surface Waves. New York: WileyInterscience.

Newman, J. N. (1976). The interaction of stationary vessels with regular waves. In Proc. 11th Symp. on Naval Hydrodynamics, London.

Srokosz, M. A. (1979). The submerged sphere as an absorber of wave power. J. Fluid Mech., 95, 717-741.

Sturova, I. V. (1994). Planar problem of hydrodynamic shaking of a submerged body in the presence of motion in a two-layered fluid. J. Applied Mechanics and Technical Physics, 35, 670-679.

Thorne, R.C. (1953). Multipole expansions in the theory of surface waves. Proc. Camb. Phil. Soc., 49, 707-716. 


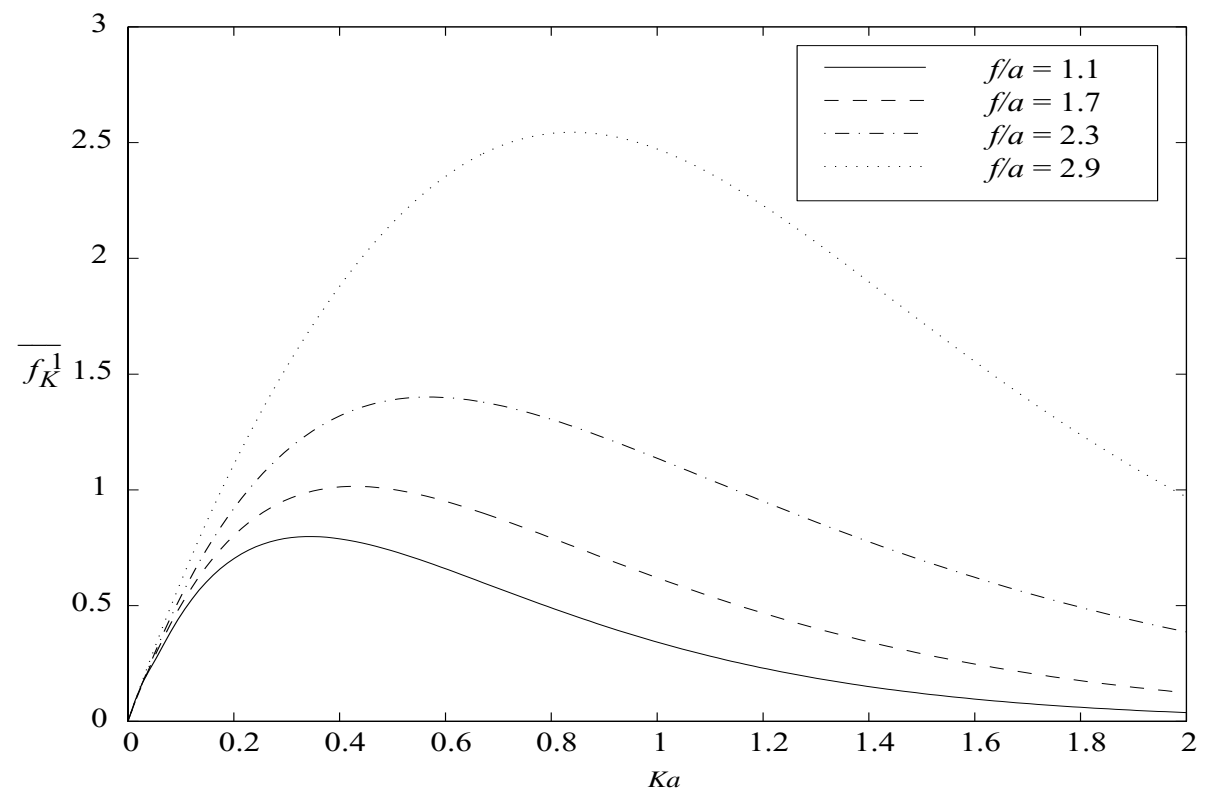

Figure 14: Non-dimensionalized horizontal exciting force $\overline{f_{K}^{1}}$ due to an incident wave of wavenumber $K$, plotted against $K a$ for different submersion depths in the upper fluid layer; $\rho=0.95$ and $d / a=4.0$.

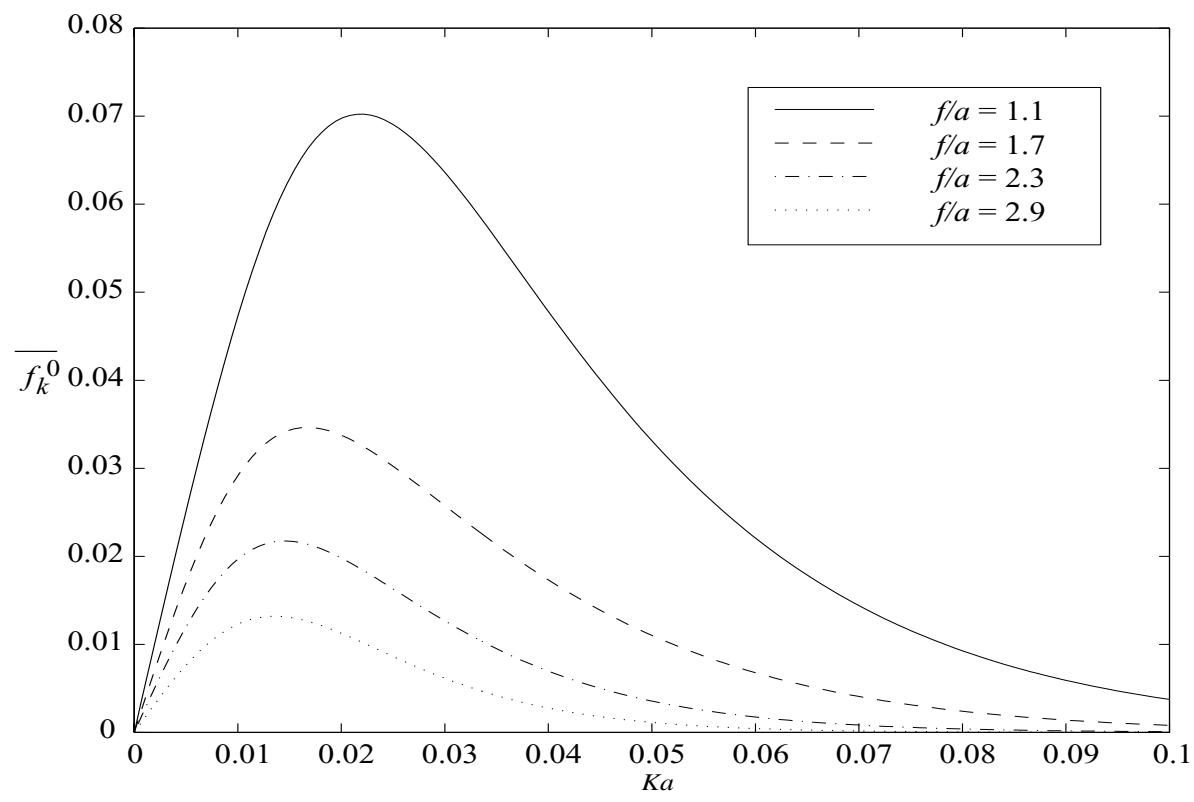

Figure 15: Non-dimensionalized vertical exciting force $\overline{f_{k}^{0}}$ due to an incident wave of wavenumber $k$, plotted against $K a$ for different submersion depths in the upper fluid layer; $\rho=0.95$ and $d / a=4.0$. 


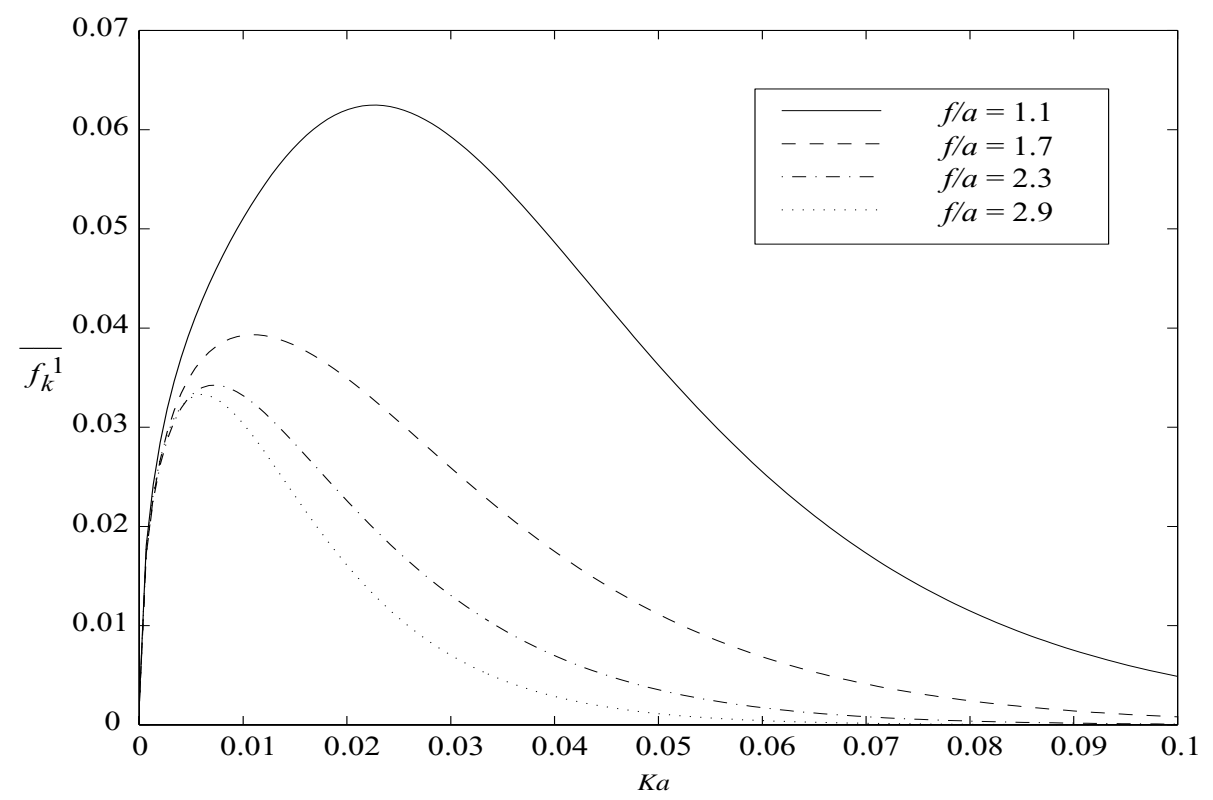

Figure 16: Non-dimensionalized horizontal exciting force $\overline{f_{k}^{1}}$ due to an incident wave of wavenumber $k$, plotted against $K a$ for different submersion depths in the upper fluid layer; $\rho=0.95$ and $d / a=4.0$. 